\title{
Collective evolution dynamics of multiple shear bands in bulk metallic glasses
}

\author{
Y. Chen, M.Q. Jiang, L.H. Dai* \\ State Key Laboratory of Nonlinear Mechanics, Institute of Mechanics, Chinese Academy of Sciences, Beijing 100190, People's Republic of China
}

\section{A R T I C L E I N F O}

\section{Article history:}

Received 12 September 2012

Received in final revised form 26 February

2013

Available online 8 April 2013

\section{Keywords:}

Bulk metallic glasses

Multiple shear bands

Size effect

Momentum diffusion

Energy dissipation

\begin{abstract}
A B S T R A C T
The collective behavior of multiple shear bands was investigated under in situ four-point bending tests of a $\mathrm{Zr}$-based bulk metallic glass (BMG) over a wide range of sample scales. The self-organization of shear band pattern, characterized by shear band spacing and shear offset, is observed with the variation of sample size and bend curvature, which presents significant size effect and tension-compression asymmetry. To unveil these fundamental behaviors, an analytical model for the evolution dynamics of multiple shear banding is developed for BMGs. In this model, both micro-structural evolution and pressure sensitivity are taken into account by introducing a new law for the stress softening of BMGs within the framework of continuum mechanics; the collective evolution of shear bands is regarded as the coupling result of the structural softening, the momentum diffusion, and the energy conservation. Applying the proposed theoretical model to the bending deformation of BMGs, the analytical solutions of shear band spacing, shear offset and failure strain are obtained. The fundamental behaviors of multiple shear bands are uncovered, in line with the experimental observations: notable scaling laws are found in the evolution of shear band spacing and shear offset, and the inhomogeneous size effect of plasticity is revealed by a transition from weak to strong size-dependence of failure strain with decreasing sample thickness. To be further, a competing map of shear band nucleation and propagation is established based on energy dissipation. The underlying mechanism of these size dependent behaviors of multiple shear bands in BMGs is found to be ascribed to the energy dissipation competition between the nucleation and propagation of shear bands.
\end{abstract}

(c) 2013 Elsevier Ltd. All rights reserved.

\section{Introduction}

Bulk metallic glasses (BMGs) constitute an emerging class of materials with excellent properties envisaged for functional and structural applications (Greer and Ma, 2007; Johnson, 1999; Schuh et al., 2007; Trexler and Thadhani, 2010; Wang, 2012). However, at temperatures well below the glass transition and at high stresses, BMGs undergo inhomogeneous deformation by concentrating severe plastic strain into nanoscale shear bands (Argon, 1979; Dai, 2012; Dai et al., 2005; Falk and Langer, 1998; Han et al., 2009; Huang et al., 2002; Jiang and Dai, 2009; Jiang et al., 2008a; Spaepen, 1977; Steif et al., 1982; Subhash and Zhang, 2007; Wu et al., 2011; Yang et al., 2005). These localized regions act as precursors to crack formation, rendering very limited ductility before catastrophic failure. Not until recently, the shear band multiplication has triggered increasingly intense scientific and technological interest of scientists, due to its remarkable enhancement of plasticity (Conner et al., 2003; Cui et al., 2010; Jiang et al., 2008c; Liu et al., 2005b; Miracle et al., 2011; Ravichandran and Molinari,

\footnotetext{
* Corresponding author.

E-mail address: lhdai@lnm.imech.ac.cn (L.H. Dai).
} 
2005; Ruan et al., 2011; Sun et al., 2010a; Zhang et al., 2008). Up to now, significant advances have been achieved to understand the nature of shear banding, however mainly focusing on the individual shear band. The fundamental problems on multiple shear banding, referring to the collective evolution dynamics and significant size sensitivity, still remain unclear.

Multiple shear bands early emerged in dynamic deformation of crystalline materials, such as explosion (Meyers et al., 2001; Nesterenko et al., 1998; Xue et al., 2002), impact (Meyers et al., 2003) and high-speed machining (Burns and Davis, 2002; Molinari et al., 2002; Ye et al., 2013). The evolution of multiple shear bands presents the features of self-organization, revealed by their characteristic periodic spacing (Meyers et al., 2001; Meyers et al., 2003; Nesterenko et al., 1998; Xue et al., 2002, 2004). In BMGs, shear band multiplication can be realized by introducing structural heterogeneity (Chen et al., 2008; Das et al., 2005; Hays et al., 2000; Hofmann et al., 2008; Liu et al., 2007; Yao et al., 2006), adopting constrained loading modes (Conner et al., 2003; Cui et al., 2010; Jiang et al., 2008c; Liu et al., 2005b; Ravichandran and Molinari, 2005; Xie and George, 2008; Yang et al., 2005; Zhang et al., 2005), or controlling the sample size and the machine stiffness (Bharathula et al., 2010; Cheng et al., 2009; Han et al., 2009). In the multiplication process, both shear band nucleation and growth are involved. The former triggers the production of new shear bands, concerned with the shear band direction (Gao et al., 2011) and spacing (Conner et al., 2004); the latter will speed the failure of BMGs, relating to the propagating mode (Cao et al., 2009; Jiang and Dai, 2011; Jiang et al., 2008c) and velocity of shear bands (Ruan et al., 2011; Song and Nieh, 2009; Wright et al., 2009) or the deformation accommodated (Jiang et al., 2007). The shear bands may operate in an intermittent, stick-slip mechanism with repeated cycles of initiation, propagation, and arrest, verified by typical serrations observed in the stress signal (Jiang et al., 2008b; Klaumünzer et al., 2011b; Song et al., 2008; Sun et al., 2010a). Analogous to those observed in crystalline metals, multiple sets of shear bands in BMGs are organized in characteristic patterns, where the shear band spacing and offset vary with sample dimension, global strain, strain rate, and normal stress (Conner et al., 2004; Jiang et al., 2008c; Yang et al., 2006; Zhang et al., 2008). Strong size effect has been revealed in the evolution of shear band patterns of different size samples (Conner et al., 2003; Ravichandran and Molinari, 2005). Reduction of material size decreases the shear band spacing and even causes a transition from shear localization to homogeneous deformation when the size is reduced to hundreds of nanometers (Greer and Hosson, 2011; Guo et al., 2007; Jang et al., 2011; Volkert et al., 2008; Yoo et al., 2012).

Great efforts have been made in characterizing multiple shear bands. Several prevailing theories for predicting shear band spacing have been developed, which are original for multiple thermoplastic shear bands of crystalline metals. Based on Mott's early analysis for dynamic fracture (Mott, 1947), Grady and Kipp $(1987,1992)$ developed a dynamic model for shear band spacing, where the band spacing on average is assumed to be equal to the width of an unloaded rigid region controlled by momentum diffusion. Lately, this theory has been extended by including the elastic strain energy (Grady, 2011). Considering shear bands arising from small growing disturbances in an initial homogeneous deformation, Wright and Ockendon (1996) proposed that the wavelength with the maximum rate of growth will tend to dominate and correspond to the most probable minimum spacing for shear bands. Then, Molinari (1997) modified the Wright-Ockendon (WO) model by adding strain hardening. Batra and Wei (2006) later derived analytical expressions of band spacing for general rate-hardening, strain-hardening material. Through integration along the characteristic lines, Zhou et al. (2006b) developed a numerical methodology adequate for analyzing the self-organized multiple adiabatic formation process in a thermo-visco-plastic material. They confirmed that the Grady-Kipp momentum diffusion theory is valid in predicting the mature stage of shear band spacing while the spacing between nucleating shear bands follows the perturbation theory due to Wright and Ockendon (Zhou et al., 2006a). Rather than strain/temperature perturbations or momentum diffusion, Meyers et al. (2001) proposed that microstructural inhomogeneities determine the initiation sites and thus shear band spacing. Thereafter, Xue et al. (2002) considered the shielding in the band nucleation and the competition among bands during the propagation stage by a two-dimensional model, which well explains the self-organization behavior of shear bands when they grow into two dimensional patterns. Recently, the evolution of shear band spacing and offset in BMGs was well described by considering a strain relaxation in the vicinity of the shear band (Conner et al., 2003) or through comparison of the energy dissipated along shear bands with the macroscopic dissipation (Ravichandran and Molinari, 2005). Based on momentum diffusion, Zhang et al. (2008) developed a thermo-mechanical model for the evolution of shear band spacing in BMGs under dynamic loading. It takes the normal stress dependence of yield stress and the associated viscosity change within the shear band region into account. These pioneer works provide an important foundation for a better understanding of multiple shear banding in BMGs.

Although the precise physical picture of how it originates from the internal structure remains elusive, it is well accepted that the shear banding of BMGs occurs as a consequence of formations and self-organizations of flow events (Chen, 2008; Schuh et al., 2007; Sun et al., 2010a; Wang, 2012). Those flow events are essentially local arrangements of atoms around free volume sites, termed shear transformation zones (STZs) or flow defects (Argon, 1979; Falk and Langer, 1998; Johnson and Samwer, 2005; Pan et al., 2008; Spaepen, 1981; Sun et al., 2010a; Sun et al., 2010b; Zhao et al., 2012). The transition from local plastic events to macroscopic shear-band instability is dominated by the stress-driven free volume softening and assisted by thermal softening (Dai et al., 2005; Gao, 2006; Gao et al., 2007; Huang et al., 2002; Jiang and Dai, 2009; Lewandowski and Greer, 2006; Yang et al., 2006; Zhang et al., 2008). Due to this micro-mechanism of inhomogeneous deformation, the macroscopic yield behavior of BMGs is found not to obey the classical pressure insensitive forms but shows a significant pressure sensitivity (Donovan, 1989; Flores and Dauskardt, 2001; Fornell et al., 2009; Hsueh et al., 2008; Ott et al., 2006; Schuh and Lund, 2003; Sun et al., 2010b). Considering the free volume caused dilatancy and pressure sensitivity, Anand and Su (2005, 2007) proposed a finite-deformation constitutive model and numerically captured major features of shear banding and 
stress-strain response of BMGs. Coupling both thermal and mechanical effects, Thamburaja and Ekambaram (2007) accurately modeled the deformation behavior of BMGs at different ambient temperatures within the supercooled liquid region. Thereafter, the sample size effect on the shear localization process in BMGs was also predicted by a non-local, thermodynamically consistent constitutive model proposed by Thamburaja (2011). The unique structural evolution induces the particular properties of shear banding in BMGs. Moreover, it is found that plastic deformation in BMGs via multiple shear bands versus a single dominant one greatly relies on the energy release during shear banding (Cheng et al., 2009; Han et al., 2009; Yang et al., 2010).

Momentum, energy and structural evolution are regarded as three fundamental factors to control the dynamics of multiple shear banding in BMGs, i.e. the nucleation of shear band (Dai, 2012; Dai et al., 2005; Jiang and Dai, 2009) and the propagation of shear band (Jiang and Dai, 2011). However, how does shear band develop into a self-organized pattern under the control of momentum, energy and structural evolution? How does material size exactly play in the dynamic deformation process? And most importantly, what are their underlying mechanisms? These critical questions have not yet been resolved. Toward this end, the authors have carried out systematic experiments of a typical Zr-based BMG under in situ four-point bending. Furthermore, a theoretical model accounting for size effect and pressure sensitivity has been developed to describe the dynamic evolution of multiple shear bands and unravel its energy mechanism. The manuscript is organized as follows: in Section 2 we briefly narrate the experimental procedure to conduct four-point bending tests on BMGs and the important features of shear band patterns. The analytical model for dynamic evolution of multiple shear bands and the solutions of the bending system are given in Section 3. Section 4 presents a detailed discussion on the evolution of shear band spacing and shear offset and its inherent scaling laws. The critical failure strain and size effect of plasticity are discussed in Section 5. Section 6 uncovers the underlying physics of multiple shear banding. In Section 7 we make salient conclusions of our present investigation.

\section{Experimental procedure and observations}

The dynamic evolution of multiple shear bands was systematically investigated under in situ four-point bending tests of a typical BMG, $\mathrm{Zr}_{41.2} \mathrm{Ti}_{13.8} \mathrm{Cu}_{12.5} \mathrm{Ni}_{10} \mathrm{Be}_{22.5}$ (Vit-1). The samples were machined into specimens (35 mm in length and $2 \mathrm{~mm}$ in width) in different thicknesses $(0.35 \mathrm{~mm}, 0.5 \mathrm{~mm}, 0.7 \mathrm{~mm}, 1.0 \mathrm{~mm}$, and $1.2 \mathrm{~mm})$. For microscopic observation, the deformed regions of the sample were polished. In situ four-point bending tests were performed with a scanning electron microscope (SEM, FEI-Sirion NC microscope) equipped with a loading stage at a velocity of $0.033 \mathrm{~mm} / \mathrm{min}$ at room temperature. The fine pattern of the shear bands was examined in situ by SEM during the bending process.

As illustrated in Fig. 1, primary shear bands nucleate at the external boundary of the specimen and propagate inside the sample, organizing a periodical distribution along a certain direction. Secondary shear bands develop along the length of the primary shear bands, particularly below the area of the shear offset. Once a shear band is activated, its propagation or slip
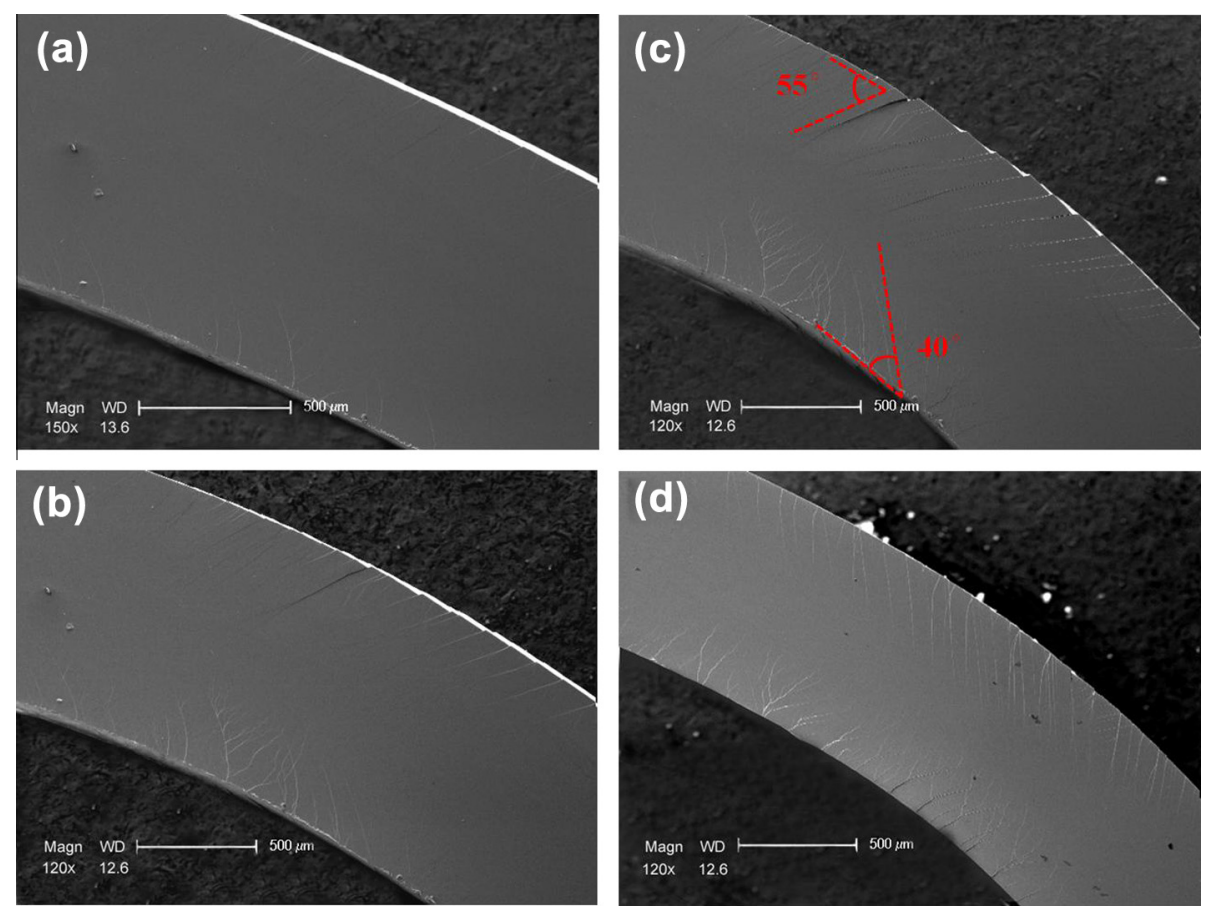

Fig. 1. SEM micrographs of Vit-1 sample under bending: $1.2 \mathrm{~mm}$ thick sample with bend radius of $15 \mathrm{~mm}$ (a), $10 \mathrm{~mm}$ (b), $5 \mathrm{~mm}$ (c); $1.0 \mathrm{~mm}$ thick sample with bend radius of $5 \mathrm{~mm}(\mathrm{~d})$. 
generates shear step (offset) with respect to its neighbors, as shown along the external boundaries. The shear band pattern evolves with the increase of the bend curvature. As an example, Fig. 1(a)-(c) shows the shear band patterns of specimens of $1.2 \mathrm{~mm}$ in thickness at different bend radii (i.e. $15 \mathrm{~mm}, 10 \mathrm{~mm}, 5 \mathrm{~mm}$ ). With increasing the bend curvature, the shear band spacing decreases first due to the appearance of new bands until a final, steady value is reached. The similar trend was also observed in Ti alloy through the radial collapse of a thick-walled cylinder (Xue et al., 2002). With bend curvature increasing, shear offset grows to be more distinct. Clearly, shear band multiplication evolves with plastic strain in company with nucleation and growth of shear bands.

The dynamic evolution of multiple shear bands is strongly size and pressure dependent. The shear band patterns for samples with different thicknesses $(1.2 \mathrm{~mm}, 1 \mathrm{~mm})$ are respectively shown in Fig. 1(c) and (d) at the same bend radius $(5 \mathrm{~mm})$. On both tensile and compressive surfaces, the shear band spacing decreases with decreasing the sample thickness, consistent with those previous experimental and simulation observations (Conner et al., 2003; Yang et al., 2006). The thinner sample usually produces denser shear bands, resulting in a better ductility. This size effect is also embodied in shear offset. Under the same bend curvature, in a thick sample shear offset is usually more significant than that in a thin sample. It implies that severer plastic deformation is accommodated by a single shear band in thicker sample, since fewer shear bands are created. To be noted, the shear band patterns on tensile and compressive surfaces are not symmetrical. The shear bands initially propagate at $\sim 55^{\circ}$ with respect to the free surface in tensile part, while in compressive part a cluster of primary shear bands with a general inclination angle of $40^{\circ}$ to the free surface is found. The asymmetrical deviation of shear band angle from $45^{\circ}$ is right consistent with that of failure angles observed in uniaxial loading (Chen et al., 2011; Jiang et al., 2008a). During the evolution, the shear band spacing and offset on the tensile side tend to be larger than those on the compressive side. It confirms the pressure-sensitivity of plastic flow in BMGs (Flores and Dauskardt, 2001; Fornell et al., 2009; Hsueh et al., 2008; Ott et al., 2006; Schuh and Lund, 2003; Sun et al., 2010b).

General features of multiple shear banding during the evolution are found, both shear band spacing and offset decrease with decreasing the sample thickness; shear band spacing decreases while shear offset increases with increasing the bend curvature. The observed shear band spacing and offset actually obey scaling laws, which will be elucidated specifically in the following discussion. In addition, the asymmetrical character of shear banding is reflected by the direction, length and density of shear bands, due to the pressure dependence of plastic deformation in BMGs.

\section{Analytical model for evolution dynamics of multiple shear banding}

Two basic processes, i.e. shear band nucleation and growth, are involved in the evolution of multiple shear banding. Nucleation takes place at the selective activation sites along certain paths; growth occurs with the competition among bands. Different from the thermoplastic shear bands in crystalline metals (Aifantis, 1987, 1995; Bai and Dodd, 1992; Batra and Lear, 2005; Chen and Batra, 1999; Dodd and Bai, 2012; Meyers, 1994; Wright, 2002; Zbib and Aifantis, 1988, 1992; Zbib and Jubran, 1992), structural or free volume softening precedes thermal softening as the origin of shear banding in BMGs (Dai, 2012; Dai and Bai, 2008; Dai et al., 2005; Gao, 2006; Gao et al., 2007; Huang et al., 2002; Jiang and Dai, 2009; Lewandowski and Greer, 2006; Thamburaja and Ekambaram, 2007; Yang et al., 2006; Zhang et al., 2008). With the new nucleation and growth of shear band, the shear band spacing and offset vary and result into different patterns as those observed in experiments. In this section, a theoretical model for the evolution dynamics of multiple shear banding is established, which analyzes the evolution of the characteristic lengths and energy dissipation of multiple shear banding in BMGs.

\subsection{Governing equations}

Complicated shear band patterns are usually found in BMGs under complex stress states, while parallel shear bands are mainly produced in unaxial or bend loading. Herein, we focus on the latter case to draw some fundamental and general understanding of multiple shear banding. As observed from our experiments, the shear bands in tensile part show a periodic and parallel distribution, although they are not strictly equally distributed. As for the compressive part, shear bands are dense and full of branches, not so regular as observed in the tensile part. The shear bands on the compressive surface usually don't present a definite angle because of the cooperative effects of material inhomogeneity and stress state. However, the primary shear bands still display a general shear direction about $40^{\circ}$ in our experiments and around $45^{\circ}$ in those of others (Conner et al., 2003; Gao et al., 2011). A typical pattern of multiple shear bands is illustrated in Fig. 2. Parallel shear bands with certain spacing $\lambda$ are distributed along a direction, as these primary shear band patterns found in compression or bending test. In this model, some reasonable assumptions are made. First, only primary shear bands are taken into account, the secondary and other shear bands which also contribute to the plastic deformation are considered to be negligible compared with the primary ones (Ravichandran and Molinari, 2005); Second, instead of every individual shear band spacing, the average shear band spacing is focused for it comprehensively represents shear band density or number. The plastic deformation accommodated or energy dissipated is dominantly determined by the number of shear band. Here we neglect the shear band difference and assume that the parallel shear bands are uniformly distributed and equivalent, analogous to those work of Conner et al. $(2003,2004)$ and Ravichandran and Molinari (2005). Since the experiments show not ideally uniform shear band patterns (see Fig. 1), average shear band spacing is calculated to enable a comparison with theory. It is worthy to note that, these assumptions will also miss some valuable information, such as the other systems of shear bands (e.g. the 


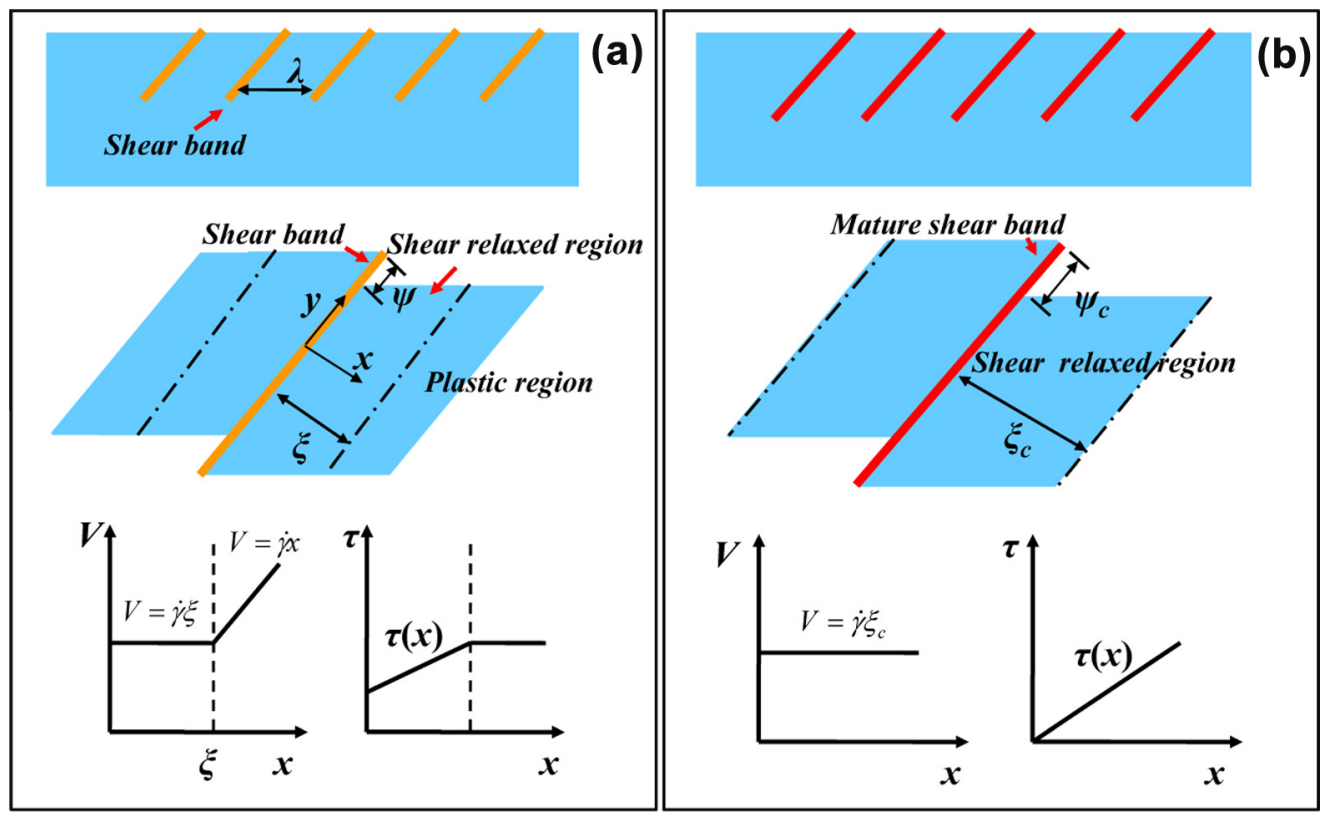

Fig. 2. Schematic of shear band evolution model and corresponding velocity and stress fields from (a) the incipience of shear band to (b) the mature stage of shear band.

secondary shear bands) and the deformation disparity in shear banding due to material inhomogeneity. In that case, possible mechanisms connecting to the microstructural inhomogeneity should be taken into account (Meyers et al., 2001; Nesterenko et al., 1998; Xue et al., 2002). In the dynamic shear band evolution, three fundamental factors govern the nucleation or propagation of shear bands in BMGs, i.e. free volume, momentum, and energy. The increment of free volume causes a decrease of viscosity, which enables the initiation of shear bands and facilitates their development; momentum diffusion adjacent to a shear band leads a stress relaxation in the layer and then produces a shield against new creation of shear bands; the whole process follows the energy conservation and the minimum energy principle.

The nucleation and coalescence of free volume decreases the flow stress of BMGs and further leads to shear localization. The material in the vicinity of the shear band unloads as momentum (velocity) diffuses. Momentum diffusion is regarded to be the governing mechanism of the shear band spacing in the evolution of multiple shear bands (Grady, 1992, 1994; Grady and Kipp, 1987; Jiang and Dai, 2011; Ye et al., 2013; Zhang et al., 2008). As illustrated in Fig. 2, a shear band residing at $x=0$ is assumed to be zero thickness due to its very small thickness compared to neighboring features (Dai and Bai, 2008; Jiang et al., 2010; Li et al., 2002). The shear band initiates along the slip plane $x=0$ at time $t=0$. The shear stress inside the shear band relaxes due to stress softening and the material unloads on both sides to a shear relaxed region of size $x= \pm \xi$ at time $t$ while in the regions $x>\xi$ and $x<-\xi$ it still remains plastic; the relaxed region moves in a uniform velocity $V$ (see Fig. 2(a)). When the shear stress inside the shear band relaxes to zero, the whole domain would relax and a mature shear band develops at a shear offset $\psi_{c}$ (see Fig. 2(b)). Material response is assumed to be rigid-plastic with the body deforming at strain rate $\dot{\gamma}$ and flow stress $\tau_{0}$. To be noted, the real material should be elastic-plastic. Provided the velocity of stress-release (even the propagation velocity of the elastic-plastic interface) is significantly lower than the elastic wave velocity (Lee, 1967), including elasticity in this analysis does not greatly alter the fluxes of energy and momentum into the shear band region, especially at strain rate higher than $10^{3} / \mathrm{s}$ (Grady, 1992; Grady, 2011). Except for the very early time response, which is elastic, the accuracy of rigid-plastic solution suffices for present study.

Corresponding to the time at which the rigid-plastic interface is at a position $\xi$, the momentum of the body per unit area can be calculated as

$$
P_{y}=\rho \dot{\gamma} \xi^{2}+\int_{\xi}^{x} \rho \dot{\gamma} x d x,
$$

where $\rho$ is the material density. The motion of the shear relaxed region can be obtained by equating the time rate of momentum in Eq. (1) to the misbalance of the shear stress as

$$
\rho \dot{\gamma} \xi \dot{\xi}=\tau_{0}-\tau,
$$

where $\tau$ is regarded as a function of the shear displacement $\psi$, the time rate of such displacement $\dot{\psi}$ and some internal state variables $I_{i}(i=1,2,3, \ldots)$, and is given as (Jiang and Dai, 2011), 


$$
\tau=\tau\left(\psi, \dot{\psi}, I_{i}\right)
$$

The shear displacement in the shear band at the boundary $x=0$ is expressed as

$$
\psi=\int_{0}^{t} \dot{\gamma} \xi d t
$$

Combining Eqs. (2)-(4) yields a system of ordinary differential equations,

$$
\begin{aligned}
& \dot{\xi}=\frac{d \xi}{d t}=\frac{\tau_{0}-\tau\left(\psi, \dot{\psi}, I_{i}\right)}{\rho \dot{\gamma} \xi}, \\
& \dot{\psi}=\frac{d \psi}{d t}=\dot{\gamma} \xi
\end{aligned}
$$

subject to the initial conditions $\xi(0)=\psi(0)=0$. The relation between $\psi$ and $\xi$ can be thus derived as

$$
\frac{d \xi}{d \psi}=\frac{\tau_{0}-\tau\left(\psi, \dot{\psi}, I_{i}\right)}{\rho \dot{\gamma}^{2} \xi^{2}} .
$$

The resolutions of $\xi$ and $\psi$ can be obtained from Eqs. (6) and (7) for a given expression of $\tau$. Considering symmetry and no interaction between adjacent shear bands, twice the distance $\xi$ will be the minimum separation (shear band spacing $\lambda$ ) between adjacent shear bands.

Assuming that the depth and the length of shear band are $b$ and $l$ respectively, the dissipation energy for an individual shear band can be expressed by

$$
E_{S B}=b l \int_{0}^{\psi} \tau d \psi .
$$

From the viewpoint of energy dissipation, the total dissipation of inhomogeneous deformation in BMGs is contributed by the primary shear bands, and the other systems (e.g. the secondary and ternary shear bands). The energy dissipated along shear bands can be equivalent to the macroscopic dissipation $W$ calculated by the conventional elasto-plastic theory, as did by Ravichandran and Molinari (2005). That is, the dissipated energy $W$ in the macro plastic region equals the total energy dissipated along shear bands including primary $E_{I}$ and others $E_{\text {oth }}$. The analysis is made for the unit length $(\Delta x=1)$, the relation can be expressed by

$$
E_{I}+E_{o t h}=W \text {. }
$$

Considering the primary shear bands play a dominant role in the whole energy dissipation, the second term in the left hand of Eq. (9) is neglected as assumed above. Therefore,

$$
E_{I} \approx W
$$

The dissipation along an individual primary shear band is measured by $E_{S B}$ and the number of bands per unit length is denoted by

$$
N_{S B}=1 / \lambda \text {. }
$$

The whole energy dissipated along the primary shear band is described as

$$
E_{I}=N_{S B} E_{S B} \text {. }
$$

The softening law of shear stress with free volume is derived by combining the micro-structural evolution with the continuum mechanics, due to the following considerations. For conventional material, there are two types of failure models. One is phenomenological and empirical, which can build a good correlation with experiments by including the effect of strain rate, temperature, hydrostatic pressure and etc. (Johnson and Cook, 1985; Khan and Liu, 2012a, b; Khan et al., 2012). The other is rooted from different physical foundations, including micro mechanically motivated failure criteria (LeRoy et al., 1981; McClintock et al., 1966; Rice and Tracey, 1969), the damage model within the framework of continuum damage mechanics (Bruhns and Schiesse, 1996; Chaboche, 1988, 2008; Lemaitre, 1985; Murakami and Ohno, 1981; Voyiadjis and Kattan, 1992, 2005), and the Gurson type model (Gurson, 1977; Needleman and Tvergaard, 1984; Wen et al., 2005). At macroscopic scale, BMGs exhibit inherent pressure sensitivity and shear-dilatancy during plastic deformation, which usually renders a non-associated flow. The yield surface of BMGs should decrease with the increase of free volume until the complete loss of load-carrying capacity. This is actually quite similar to the void evolution mechanism in the continuum mechanics. One of the best known micro-mechanical models is due to Gurson (1977) with modifications by Needleman and Tvergaard (1984), Tvergaard $(1981,1982)$, it studies the plastic flow of a void-containing material and establishes a yielding function reflecting the softening effect due to the presence of voids. In BMGs, "free volume" as the topological disorder, can be simply considered as randomly distributed atomic scale voids in material. Treating these voids to be spherical, the yield 
function of Gurson is reasonably extended to BMGs by taking into the pressure sensitivity of the matrix. Moreover, the free volume evolution is under the control of the stress-induced free volume creation and the free volume annihilation due to structural relaxation and diffusion (Dai et al., 2005; Huang et al., 2002; Jiang and Dai, 2009; Thamburaja and Ekambaram, 2007).

For a pressure-independent material containing spherical voids, the GTN model uses the yield function (Gurson, 1977; Needleman and Tvergaard, 1984)

$$
F=\left(\frac{\sigma_{e}}{\sigma_{y}}\right)^{2}+2 q_{1} v_{f} \cosh \left(\frac{3 \sigma_{m}}{2 \sigma_{y}}\right)-\left(q_{1} v_{f}\right)^{2}-1=0,
$$

where $\sigma_{e}=\sqrt{3 s_{i j} s_{i j} / 2}=\sqrt{3 J_{2}}\left(s_{i j}=\sigma_{i j}-\sigma_{m} \delta_{i j}\right)$ is the effective stress, $\sigma_{m}=\sigma_{i i} / 3$ is the mean stress, $v_{f}$ is the current fraction of voids, equivalent to free volume concentration, $\sigma_{y}$ is the yield stress of the matrix, and $q_{1}$ is a constant introduced by Tvergaard $(1981,1982)$. When the void fraction reaches $1 / q_{1}$, material will lose load carrying capacity. The above criterion can be rewritten as below

$$
\left(\frac{\sigma_{e}}{\sigma_{y}}\right)^{2}=\left(1-q_{1} v_{f} \cosh \left(\frac{3 \sigma_{m}}{2 \sigma_{y}}\right)\right)^{2}-\left(\cosh ^{2}\left(\frac{3 \sigma_{m}}{2 \sigma_{y}}\right)-1\right)\left(q_{1} v_{f}\right)^{2} .
$$

For simplicity, we neglect the minor term $\left(\cosh ^{2}\left(3 \sigma_{m} / 2 \sigma_{y}\right)-1\right)\left(q_{1} v_{f}\right)^{2}$ in Eq. (14), as $v_{f} \ll 1$ for BMGs. Note that the original GTN model considers the von Mises matrix. The macroscopic pressure sensitivity of yielding comes from the pressure dependence of voids. However, the pressure-sensitive yielding has also been found in the matrices of porous materials (Jeong and Pan, 1995; Lazzeri and Bucknall, 1993). BMGs can be considered as a void-containing material by treating free volume as randomly distributed atomic scale voids. On the one hand, the free volume presents pressure dependence as traditional voids or cavities, from the viewpoint of geometry; on the other hand, the yielding of "matrix' (excluding the geometrical free volume) is also pressure-dependent due to its topological disorder. In crystalline solids, unit glide of a dislocation does not require significant dilatation. Due to the long-range disorder structure, BMGs cannot find a slip plane when they experience shear deformation. As a result, the plastic flow units in BMGs (i.e. shear transformation zones) change into a loose configuration with a considerable dilatation (Dyre et al., 1996; Jiang and Dai, 2007). The inherent dilatation is found to be the major reason of the pressure sensitivity of plastic flow in BMGs (Schuh et al., 2007; Sun et al., 2010b). In order to involve this pressure sensitivity of flow, we introduce an additional pressure-dependent term i.e. $\alpha \sigma_{m}$, into Eq. (14) for a proper description of BMGs. The yield criterion is therefore established in the form of

$$
\Phi=\sqrt{J_{2}}+\alpha \sigma_{m}-\frac{\sigma_{y}}{\sqrt{3}}\left(1-q_{1} v_{f} \cosh \left(\frac{3 \sigma_{m}}{2 \sigma_{y}}\right)\right)=0
$$

where $\alpha$ is the pressure sensitive factor. This new criterion (15) is analogous to the Drucker-Prager criterion, but takes the free volume softening into account. We define the initial free volume in BMGs to be $v_{0}=\left[T-T_{\text {ref }}+\sqrt{\left(T-T_{\text {ref }}\right)^{2}+d_{2} T}\right] / 2 d_{1}$ utilizing the Cohen-Grest model (Grest and Cohen, 1981; Yang et al., 2006; Zhang et al., 2008), where $T$ is the initial temperature, $d_{1}, d_{2}$, and $T_{\text {ref }}$ are material parameters. The shear strength for $v_{f}=v_{0}$ is then calculated to be $\sigma_{0}=\sigma_{y}\left(1-q_{1} v_{0} \cosh \left(3 \sigma_{m} / 2 \sigma_{y}\right)\right) / \sqrt{3}$. So, Eq. (15) can be written as

$$
\Phi=\sqrt{J_{2}}+\alpha \sigma_{m}-\sigma_{0}(1-\beta \Lambda) \text {, }
$$

where $\Lambda=v_{f}-v_{0}$ is the free volume increment, and the free volume softening coefficient $\beta=1 /\left(\operatorname{sech}\left(3 \sigma_{m} / 2 \sigma_{y}\right) / q_{1}-v_{0}\right)$.

The free volume evolution within the shear band can be expressed as the following equation (Dai et al., 2005; Huang et al., 2002; Jiang and Dai, 2009; Thamburaja and Ekambaram, 2007),

$$
\dot{v}_{f}=D_{f} \nabla^{2} v_{f}+\zeta
$$

where $D_{f}$ is the diffusion coefficient of free-volume concentration and $\zeta$ is the net generation rate of free volume. According to the self-consistent dynamic free volume model (Johnson et al., 2002), the net generation rate of the free volume is $\zeta=\varpi\left(v_{R} \dot{\varepsilon}_{e}^{p}-\Lambda /\left(\sigma_{e} /\left(G \dot{\varepsilon}_{e}^{p}\right)\right)\right)$, where $\varpi$ is a material parameter of order unity, $v_{R}$ is a free-volume creation function defining the free volume produced by a unit shear strain and is given by $G \Lambda / \sigma_{s s}\left(\sigma_{s s}\right.$ is the effective stress at steady state) (Yang et al., 2006), $\dot{\varepsilon}_{e}^{p}=\sqrt{2 \dot{e}_{i j}^{p} \dot{e}_{i j}^{p} / 3}\left(\dot{e}_{i j}^{p}=\dot{\varepsilon}_{i j}^{p}-\dot{\varepsilon}_{k k}^{p} \delta_{i j} / 3\right)$ is the effective plastic shear strain rate, $\sigma_{e}$ is the effective stress, and $G$ is the shear modulus at room temperature. The first term in the right hand side, $v_{R} \dot{\varepsilon}_{e}^{p}$, assumes that the free volume creation is proportional to the plastic strain rate, whereas the relaxation process is described by the second term.

The free-volume softening within the shear band leads to a particular stress-displacement behavior, which can be assumed as a linear relationship for the release of shear stress with increasing shear displacement for simplicity (Grady, 1992; Ye et al., 2013; Zhang et al., 2008),

$$
\tau(\psi)=\tau_{0}\left(1-\frac{\psi}{\psi_{c}}\right)
$$

where the critical shear offset $\psi_{c}$ is required to form a mature shear band, ahead of which the stress relaxation is related to the shear displacement with the softening law (16) expending a finite amount of energy in the process. When it is reached, the shear stress within shear band reduces to zero. Combining Eqs. (16) and (18), we can derive that $\psi=\beta \Lambda \psi_{c}$. Here $\beta \Lambda$ mea- 
sures the evolution degree of shear band. In fact, as to the multiple shear banding of crystalline metals in high speed machining (Molinari et al., 2002; Ye et al., 2013), an index for the evolution degree of shear band has also been found (Ye et al., 2013). According to Eq. (8), the dissipated energy $E_{S B}$ is obtained as

$$
E_{S B}=b l \int_{0}^{\psi} \tau_{0}\left(1-\frac{\psi}{\psi_{c}}\right) d \psi=b l(1-\beta \Lambda / 2) \beta \Lambda \tau_{0} \psi_{c} .
$$

Introducing Eq. (19) into Eq. (12), we have

$$
E_{I}=\frac{b l(1-\beta \Lambda / 2) \beta \Lambda \tau_{0} \psi_{c}}{\lambda} .
$$

On the other hand, applying Eq. (18) into Eq. (7), we can derive that

$$
\xi^{3}=\frac{3}{2} \frac{\tau_{0}}{\rho \dot{\gamma}^{2}} \frac{\psi^{2}}{\psi_{c}} .
$$

Introducing Eq. (21) into Eq. (6), the expression for $\psi$ is obtained as

$$
\psi=\frac{1}{18} \frac{\dot{\gamma} \tau_{0}}{\rho} \frac{t^{3}}{\psi_{c}},
$$

and then $\xi$ is expressed by

$$
\xi=\frac{1}{6} \frac{\tau_{0}}{\rho} \frac{t^{2}}{\psi_{c}}
$$

At the time $t$, shear band slips a displacement of $\psi$ and the momentum diffuses to a distance of $\xi$. When $\beta \Lambda=1$, indicative of a zero stress within shear band, $t$ reaches a critical time $t_{c}$ and the shear offset develops to $\psi_{c}$. Considering symmetry and no interaction between adjacent shear bands, the shear band spacing $\lambda$, as the minimum separation between adjacent shear bands, can be estimated by $2 \xi / \sin \theta$. Thus, using Eqs. (22) and (23) and $\psi=\beta \Lambda \psi_{c}$, the shear band spacing can be derived as

$$
\lambda=\left(\frac{12 \beta^{2} \Lambda^{2} \psi_{c} \tau_{0}}{\rho \dot{\gamma}^{2}}\right)^{1 / 3} / \sin \theta .
$$

Combining Eqs. (10), (20), and (24), we obtain the shear band spacing and offset, respectively, as

$$
\begin{aligned}
& \lambda=\sqrt{\frac{12 \beta \Lambda W}{b l \rho(1-\beta \Lambda / 2) \dot{\gamma}^{2} \sin ^{3} \theta}}, \\
& \psi=\sqrt{\frac{12 \beta \Lambda W^{3}}{b^{3} l^{3} \rho(1-\beta \Lambda / 2)^{3} \tau_{0}^{2} \dot{\gamma}^{2} \sin ^{3} \theta}},
\end{aligned}
$$

where the shear band spacing and the shear offset are related to the free-volume softening factor $\beta \Lambda$, the dissipated energy $W$, and the strain rate $\dot{\gamma}$. The shear angle $\theta$ is also included in the analytical expressions. Its asymmetry between tension and compression would cause the asymmetrical evolution of shear band spacing and shear offset. The critical shear offset is then obtained in the form of $\psi_{c}=\sqrt{96 W_{c}^{3} / b^{3} l^{3} \rho \tau_{0}^{2} \dot{\gamma}^{2} \sin ^{3} \theta}$ by setting $\beta \Lambda=1$ and $W=W_{c}$. $W_{c}$ is the energy dissipation of mature shear bands. The present model can investigate multiple shear banding for BMGs under a range of constrained loading conditions. To be noted, the macroscopic dissipations $W$ and $W_{c}$ depend on specific configuration, involved with material dimension and loading state. Analytical solutions of them can be derived in some simple stress states (i.e. unaxial loading or bending), but in the other complicate situations (i.e. indentation), it may be difficult to estimate. Next, the behavior of multiple shear bands in a typical bending deformation of BMG is discussed by applying this model, the analytical solutions for shear band spacing, shear offset and energy dissipation are obtained.

\subsection{Solutions of the bending system}

In the above model (Section 3.1), the macroscopic dissipation $W$ relies on specific material and loading condition. According to the four-point bending experiments, plane strain conditions prevail for thin plates. We consider an initially straight beam of unit length and rectangular cross-section with thickness $2 h$, and width $b$, as illustrated in Fig. 3 . It is recognized that the shear bands initiate at the external boundaries of the beam and propagate in the plastically deforming region. Parallel shear bands grow inwards towards the neutral axis at an angle $\theta^{T}$ or $\theta^{C}$ and terminate at the elastic plastic boundary. The shear bands on tensile side of the beam exhibit different spacing and inclination angles from those on compressive side, as observed in the current experiments. A typical shear band in a beam under bending is shown in Fig. 3, with the associated coordinate system, $x_{1}-x_{2}$ ( $x_{1}$-axis being in the direction along the band and $x_{2}$-axis being normal to the band). The spacing 


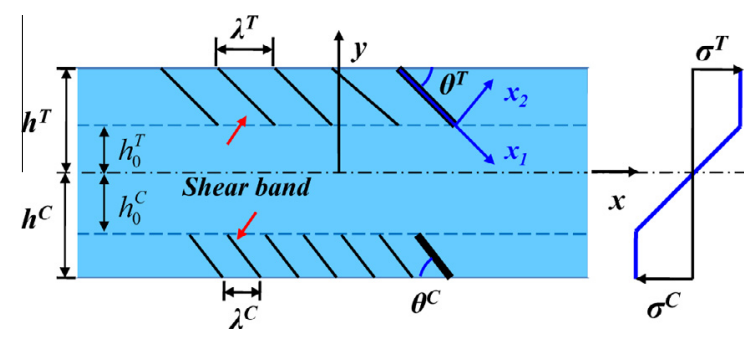

Fig. 3. Schematic of the primary shear band pattern with uniform spacing $\lambda^{T}$ and inclination angle $\theta^{T}$ on tensile side and $\lambda^{C}$ and $\theta^{C}$ on compressive side. Stress state in the cross-section of the beam beyond yield is illustrated.

$\lambda^{T}$ on tensile side and $\lambda^{C}$ on compressive side of the primary shear bands are taken as uniform. $\psi^{T}$ and $\psi^{C}$ are the slip displacements due to plastic shearing along a shear band.

One-dimensional Bernoulli-Euler beam theory is used here. Plane cross-sections remain plane and normal to neutral axis remain normal during deformation. The small strain theory is employed. Due to lacking strain hardening capability, BMGs are assumed to be elastic-perfectly plastic. Assuming the stress-free boundaries conditions at $y=-h^{C}$ and $y=h^{T}$ and the plane strain state, the components of the stress and strain tensors in the frame $O x y z$ of Fig. 3, satisfy $\sigma_{y y}=0, \sigma_{z z}=v \sigma_{x x}$, $\sigma_{i j}=0(i \neq j)$ and $\varepsilon_{z z}=\varepsilon_{i j}=0(i \neq j)$. According to the yield criterion (see Eq. (16)), the critical stresses on tensile and compressive sides to cause material yielding are respectively

$$
\begin{aligned}
& \sigma_{x x}^{T}=\sigma_{s} / D^{T}, \\
& \sigma_{x x}^{C}=-\sigma_{s} / D^{C},
\end{aligned}
$$

where the yield stress $\sigma_{s}=\sigma_{0}(1-\beta \Lambda), D^{T}=\sqrt{\left(1+v^{2}-v\right) / 3}+\alpha(1+v) / 3$ and $D^{C}=\sqrt{\left(1+v^{2}-v\right) / 3}-\alpha(1+v) / 3$. In the elementary beam theory, the total axial strain $\varepsilon_{x x}$ varies linearly in the thickness direction $y$, i.e. $\varepsilon_{x x}=y / R=k y$, where $R$ and $k$ are respectively the bend radius and the bend curvature. Because $\sigma_{x x}=E^{\prime} \varepsilon_{x x}=E^{\prime} k y\left(E^{\prime}=E /\left(1-v^{2}\right)\right)$, we can conclude the location of the elastic-plastic boundary both on tensile and compressive sides, respectively, as $h_{0}^{T}=\sigma_{s} / k E^{\prime} D^{T}$ and $h_{0}^{C}=\sigma_{s} / k E^{\prime} D^{C}$. Note that the regions, $y \leqslant-h_{0}^{C}$ and $y \geqslant h_{0}^{T}$, are the "plastic zones" of the beam. Because of the pressure sensitivity, the beam experiences non-symmetric bending beyond yield. The neutral axis $y=0$ does not situate in the middle of the sample, and it can be found $h^{T}=\kappa h^{C}-(\kappa-1 / \kappa) h_{0}^{C} / 2\left(\kappa=D^{T} / D^{C}\right)$ by requiring the total in-plane force acting in the beam to be zero. Using the stress-strain relation, the strain within the plastic regions can be obtained, including the elastic term and the plastic term, i.e. $\varepsilon_{x x}=\sigma_{s} / D^{T} E^{\prime}+\varepsilon_{x x}^{p}$ for $h_{0}^{T} \leqslant y \leqslant h^{T}$ and $\varepsilon_{x x}=-\sigma_{s} / D^{C} E^{\prime}+\varepsilon_{x x}^{p}$ for $-h^{C} \leqslant y \leqslant-h_{0}^{C}$, where $\varepsilon_{x x}^{p}$ is the plastic strain parallel to the $x$-axis.

The shear stress within the shear band can be related to the normal stress given by the beam theory,

$$
\tau^{*}=\sigma_{x x}^{*} \cos \theta^{*}=\tau_{0}^{*}\left(1-\beta \Lambda^{*}\right),
$$

where the superscript "*" denotes "T" on tensile side or "C" on compressive side, the critical shear stress $\tau_{0}^{*}=\sigma_{0} \cos \theta^{*} / D^{*}$. From the macroscopic point of view, the total dissipation may be evaluated by considering the plastic beam theory. According to this theory, the deformation in the plastic region is regarded to be homogenous and the free-volume softening is far smaller than that within the localized shear bands. Therefore, $\Lambda$ within the plastic region is ignorable and $\sigma_{s} \approx \sigma_{0}$. Considering the plastic work is entirely dissipated, the dissipation in the plastic region is

$$
W=\int_{y \geqslant 0 \text { or } y \leqslant 0} \sigma_{x x}^{*} \varepsilon_{x x}^{p} d V=b \frac{\sigma_{0}}{D^{*}} \int_{h_{0}^{*}}^{h^{*}} \varepsilon_{x x}^{p}(y) d y=\frac{b k\left(h^{*}-h_{0}^{*}\right)^{2} \sigma_{0}}{2 D^{*}} .
$$

We note that, both elastic and plastic responses are considered in the bending deformation, whereas, a rigid-plastic response is assumed in the momentum diffusion analysis as discussed before. These two are actually not conflicted in the present study. First, momentum diffusion plays effect in the deformation stage when material already yields and experiences considerable plastic flow, and the elastic deformation becomes a minor term. Second, the fluxes of energy and momentum into the shear band should not be markedly altered by including the elasticity when the velocity of stress-release is significantly lower than the elastic wave velocity. In this case, the elastic response can be neglected in the momentum diffusion. An energy balance is then established by assuming that energy dissipated by plastic deformation in pure bending is equal to that dissipated by shear bands. Applying Eq. (29) and $l^{*}=\left(h^{*}-h_{0}^{*}\right) / \sin \theta^{*}$ into Eqs. (25) and (26), we obtain the shear band spacing and offset in tensile part and compressive part, respectively, as

$$
\lambda^{*}=\sqrt{\frac{6 \sigma_{0} \beta \Lambda^{*} k\left(h^{*}-h_{0}^{*}\right)}{\rho\left(1-\beta \Lambda^{*} / 2\right) D^{*} \sin ^{2} \theta^{*} \dot{\gamma}^{2}}},
$$




$$
\psi^{*}=\sqrt{\frac{3 \sigma_{0} \beta \Lambda^{*}\left(k\left(h^{*}-h_{0}^{*}\right)\right)^{3}}{2 \rho\left(1-\beta \Lambda^{*} / 2\right)^{3} D^{*} \cos ^{2} \theta^{*} \dot{\gamma}^{2}}} .
$$

These length scales rely on material properties, sample size, bend curvature, and strain rate as well. Usually, the local strain rate $\dot{\gamma}$ is hard to be obtained directly from experiments. For quasi-static bending test, the strain rate depends on the material parameters and loading conditions $(k, \dot{k})$. Since material parameters are constant for a given material, the strain rate can be expressed as $\dot{\gamma}=f(\dot{k} / k)$ through dimensional analysis. For simplicity, it is supposed $\dot{\gamma}$ as a linear function of $\dot{k} / k$. It can be further derived that $\dot{k} / k=-k d R / d t$, where $d R / d t$ is the time rate of the bend radius and is constant in the current experiments. Therefore, the strain rate can be given as $\dot{\gamma}=\chi k$, where $\chi$ is a proportionality parameter only depending on material properties. In the following, we have a detailed discussion on the evolution behavior of multiple shear bands in BMGs under bending, and a comparison between theory and experiments is made.

\section{Evolution of shear band spacing and shear offset and its scaling laws}

In this section, the close dependence of shear band spacing and shear offset on sample thickness, bend curvature, and further the bending ratio (thickness versus bend radius), obeying notable scaling laws, for Vit-1 BMG is discussed both from theoretical analysis and experiments. What's else, the obvious tension-compression asymmetry due to pressure sensitivity is described. The mechanical and material constants for Vit-1 BMG are given as (Lu et al., 2003; Yang et al., 2006): $E=96 \mathrm{GPa}, \sigma_{0}=1 \mathrm{GPa}, \rho=6120 \mathrm{~kg} / \mathrm{m}^{3}, v=0.36, \alpha=0.1$, and $h^{T}=h^{C}=h$ can be approximated. To determine the factor $\chi$, we introduce a typical group of experimental data into Eq. (30): $h=0.5 \mathrm{~mm}, k=1 / 5 \mathrm{~mm}^{-1}, \lambda^{T}=68 \mu \mathrm{m}, \theta^{T}=55^{\circ}$, $\lambda^{C}=65 \mu \mathrm{m}, \quad \theta^{C}=40^{\circ}$. Then it is obtained that $\beta \Lambda^{T} /\left[\left(1-\beta \Lambda^{T} / 2\right) \chi^{2}\right] \approx 8.4 \times 10^{-10}\left(\mathrm{~s}^{2} / \mathrm{m}^{2}\right)$ and $\beta \Lambda^{C} /\left[\left(1-\beta \Lambda^{C} / 2\right) \chi^{2}\right] \approx 4.2 \times 10^{-10}\left(\mathrm{~s}^{2} / \mathrm{m}^{2}\right) . \Lambda^{T}$ is usually larger than $\Lambda^{C}$, and the both are reasonably set of the order $\sim 10^{-3}$ during the steady plastic flow (Yang et al., 2006). $\beta$ is estimated to be $\sim 10^{2}$ as shear resistance is completely lost when $\Lambda \sim 10^{-2}$. $\chi$ is then educed to be of the order $\sim 10^{4} \mathrm{~m} / \mathrm{s}$. The local strain rate $\dot{\gamma}$ is calculated to be $\sim 10^{6} \mathrm{~s}{ }^{-1}$, within the scope of local strain rate $10^{3} \sim 10^{9} \mathrm{~s}^{-1}$ encompassing the macroscopic loading rates from quasi-static to dynamic range (Zhang et al., 2008). These results will be utilized for the following discussion.

Fig. 4(a) and (b) demonstrates shear band spacing as a function of bend curvature for Vit- 1 samples of constant thickness $(0.35,0.5,0.7,1.0$, and $1.2 \mathrm{~mm})$, both for tensile and compressive sides. A common trend is observed for all the curves. The initial shear band spacing does not emerge until a critical bend curvature is reached. At this bend curvature, material yields and the inhomogeneous deformation via shear banding occurs. At the beginning, the shear bands usually have a maximum spacing to each other due to the least shear bands nucleated. With the increase of bend curvature, new shear bands would appear and a decrease of shear band spacing follows. To be noted, the shear band spacing changes more dramatically at the initial stage, which suggests shear band prefers to nucleate at this period. With decreasing sample thickness, the shear band spacing decreases and the slopes of curves become flatter gradually. We can see that the thinner sample always corresponds to a smaller spacing, indicating a greater number of shear bands or a better capability of shear band multiplication. These theoretical predictions capture well the experimental results as marked by open symbols. These experimental data of shear band spacing were measured by averaging the number of shear bands over the same length. This decrease in the shear band spacing with increasing plastic strain was also reported by Bei et al. (2006) through various amounts of compression on Zr-based BMGs. Meanwhile, the opposite change trend of spacing with bend curvature, i.e., the shear band spacing steadily increases with increasing bend curvature for constant sample thickness, was observed by Conner et al. (2004). The corresponding theories were established to describe this phenomenon (Conner et al., 2003, 2004; Ravichandran and Molinari, 2005). There are two possible reasons to cause these distinct results. First, the extension in the tensile part would enlarge shear band spacing when no new shear bands come out, this effect is especially significant at large bend curvature. We see that the bend curvatures of Conner et al. (2003, 2004) (i.e. $1 / 3 \mathrm{~mm}^{-1} \sim 2 \mathrm{~mm}^{-1}$ ) are generally bigger than those of our experiments (i.e. $1 / 15 \mathrm{~mm}^{-1} \sim 1 / 3 \mathrm{~mm}^{-1}$ ); Second, new shear bands may be inclined to nucleate at the initial stage or at a relatively small bend curvature, as discussed above. That is, shear band density probably increases greatly at the beginning but hardly changes after a certain bend curvature. The both change trends of spacing with global strain were captured by Meyers and co-workers (Xu and Meyers, 2012; Xue et al., 2002) for titanium and Ti alloy. They attributed the increase of shear band spacing to the dead shear bands which may be merged into the large plastic deformation of surrounding area. However, this annihilation of shear bands in BMGs is not observed in the current experiments. Despite the similar dependence on bend curvature, the shear band spacing on the compressive side (Fig. 4(b)) is relatively smaller than that on the tensile side (Fig. 4(a)).

As widely reported (Chen and Lin, 2010; Conner et al., 2004; Ravichandran and Molinari, 2005), the shear band spacing in constrained geometries is proportional to a characteristic sample dimension. The constant of proportionality may depend on material composition (Cui et al., 2010) and loading state (Sergueeva et al., 2005; Subhash and Zhang, 2007). The shear band spacing is plotted as a function of sample thickness in Fig. 5, for tensile and compressive sides respectively. Fig. 5(a) shows that shear band spacing enlarges with increasing sample thickness under different curvature radii ( $3 \sim 15 \mathrm{~mm}$ ). A scaling law is found that the shear band spacing varies directly as the square root of the thickness for a given bend radius. Take the red solid curve (under bend radius of $15 \mathrm{~mm}$ ) for example, the shear band spacing decreases slowly with the reduction of sample thickness at first. However, when the thickness comes close to 0.5 mm, the shear 
(a)

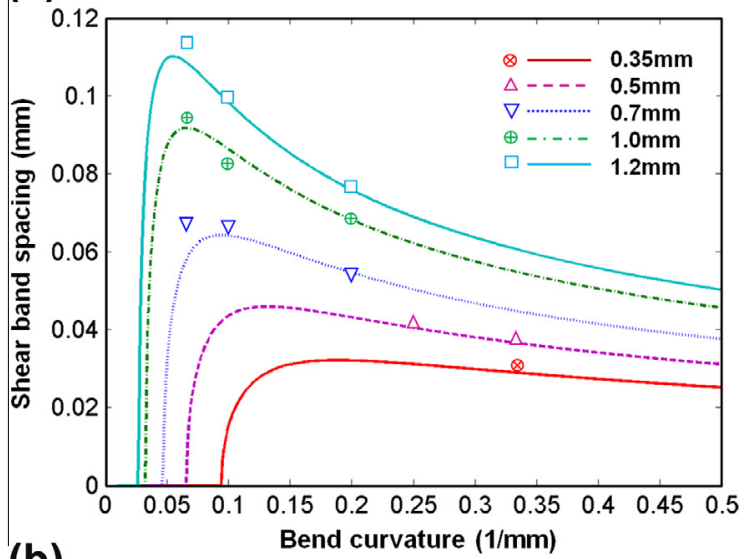

(b)

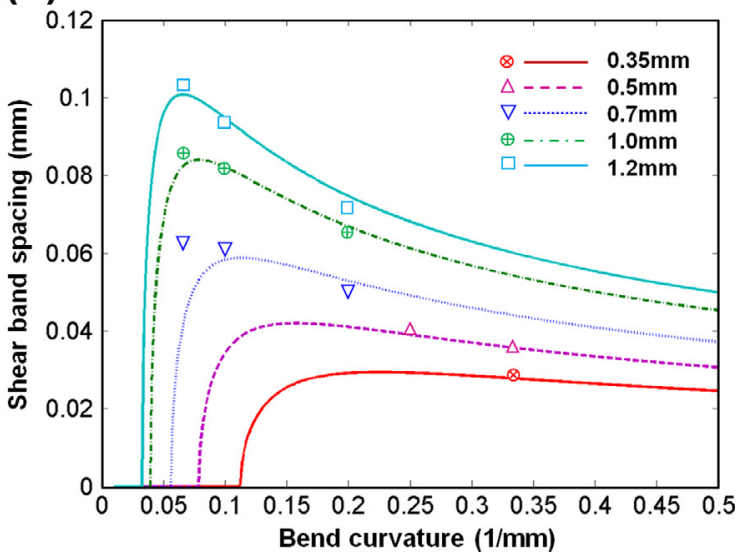

Fig. 4. Shear band spacing as a function of bend curvature under different sample thicknesses, (a) for tensile side and (b) for compressive side. The unconnected symbols denote the average shear band spacing derived from the current experiments.

band spacing drops very quickly. This trend indicates that the size effect of shear band spacing is not homogeneous, although it is generally reasonable to take shear band spacing to be $\sim 1 / 10$ of a dimension, as first reported by Conner et al. (2003, 2004). The quick drop of the shear band spacing implies that a large number of shear bands can emerge below a critical thickness, in other words, a homogeneous plastic deformation can be expected when a sample is small enough. Indeed, this has been realized in the compression (Greer and Hosson, 2011; Jang et al., 2011; Volkert et al., 2008) and tension (Guo et al., 2007) of BMG nano-pillars. The experimental data for Vit-1 are plotted as open symbols. Apparently, the observed shear band spacing agrees well with the theoretical prediction. Fig. 5(b) plots shear band spacing versus thickness on the compressive side. These shear bands in compression show a similar change in spacing with sample thickness. However, the shear band spacing on the compressive side is generally smaller than that on the tensile side under the same condition, in line with those observed in experiments. It is known that shear banding is a mechanism of inelastic deformation taking place within the plastic zone. The size of plastic zone should therefore closely relate to the shear band spacing. As seen in Eq. (30), the spacing is scaled with the plastic zone size $h^{T}-h_{0}^{T}$ on tensile side and $h^{C}-h_{0}^{C}$ on compressive side. Attributed to the pressure sensitivity, $h^{T}-h_{0}^{T}$ is usually larger than $h^{C}-h_{0}^{C}$, which thus leads to a wider spacing in tensile part than that in compressive part.

Shear offset can range from several nanometers to around hundred microns depending on sample dimension and loading condition (Conner et al., 2004; Flores and Dauskardt, 2006; Kim et al., 2002; Wright et al., 2001). Under quasi-static bending, the variation of the shear offset with bend curvature on the tensile side for different thicknesses $(0.35,0.5,0.7,1.0$, and $1.2 \mathrm{~mm}$ ) is shown in Fig. 6(a). We note that the shear slip is activated when a critical bend curvature is reached, analogous to those found in shear band spacing. The thicker sample is, the bigger bend curvature is required to create an initial shear displacement or trigger a shear band. Fig. 6(a) also describes that, with the increase of bend curvature, the shear offset grows. Moreover, it grows more slowly in a thinner sample than that in a thicker sample. This implies that single shear band would propagate faster in a thick sample and easily form crack. The shear offset on the tensile side for a given curvature radius $(3,5,10,15 \mathrm{~mm})$ for beams of varying thickness is shown in Fig. 6(b). The shear offset varies with the three-seconds power 
(a)
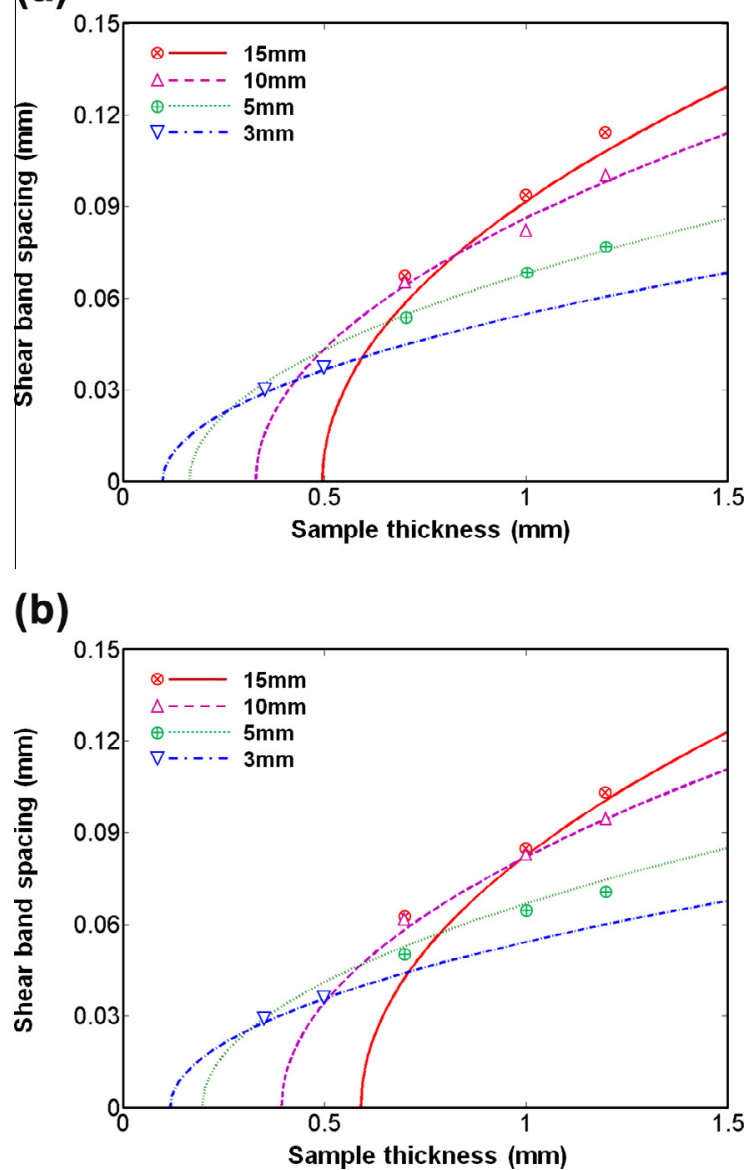

Fig. 5. Shear band spacing as a function of sample thickness under different bend radii, (a) for tensile side and (b) for compressive side. The theoretical prediction shows a good agreement with the experimental results (marked by unconnected symbols).

of the thickness and it can range from nanometers to microns for Vit-1 BMGs. The magnitude of shear offset reflects the plastic deformation contained by a shear band. Usually, the big shear offset, indicative of highly localized strain, is adverse to macro-plasticity.

To be further, the expression of shear band spacing and shear offset (Eqs. (30) and (31)) can be normalized. Then, the dimensionless shear band spacing $\lambda^{*} / R$ and shear offset $\psi^{*} / R$ can be expressed as the functions of the bending ratio $h^{*} / R$. The general dependence of shear band spacing and shear offset on bending ratio can be therefore obtained, as depicted in Fig. 7, both for tensile and compressive parts. This helps us to have an overall understanding of the coupling effect of sample thickness and bending curvature on multiple shear banding. Shear band spacing and shear offset are positive only when the bending ratio or macro-strain is above a critical value for material yielding, as discussed previously. The increase trend with bending ratio is both found in the normalized shear band spacing and offset. We note that, the non-normalized shear band spacing can show an opposite trend since it is increased with increasing sample thickness but decreasing bend curvature. The tension-compression asymmetries of shear band spacing and shear offset are obviously reflected. In contrast to the minor asymmetry of shear band spacing, it can be found that this asymmetry of shear offset becomes stronger with increasing bending ratio. The shear bands in tensile part normally propagate faster than those in compressive part due to the opposite constraints. This priority is particularly true with the increase of bending ratio, which tells that the cracking of BMGs commonly initiates from the tensile part.

\section{Critical strain at fracture and size effect of plasticity}

From the evolution of shear band spacing and shear offset, as discussed in the above section, we estimate the plastic capability of BMGs indirectly. In this part, the macro-plasticity will be judged directly by a critical strain at which cracks initiate and result in material fracture. As mentioned previously, the shear band develops into a mature one through stress softening 
(a)

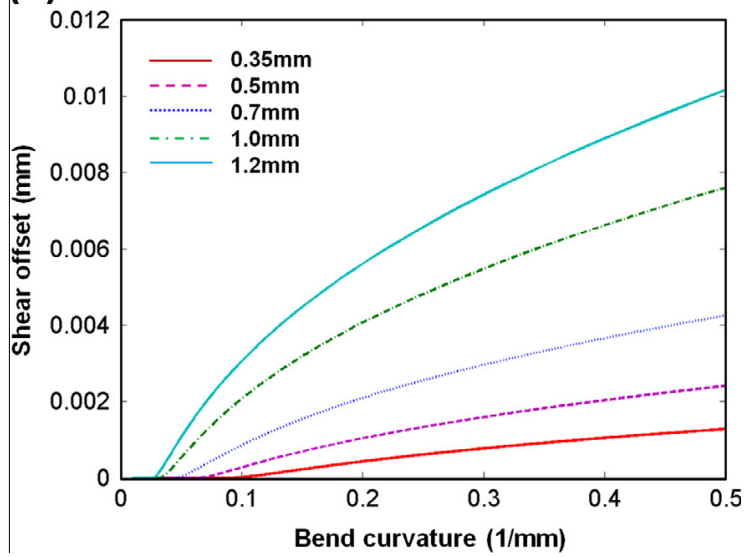

(b)

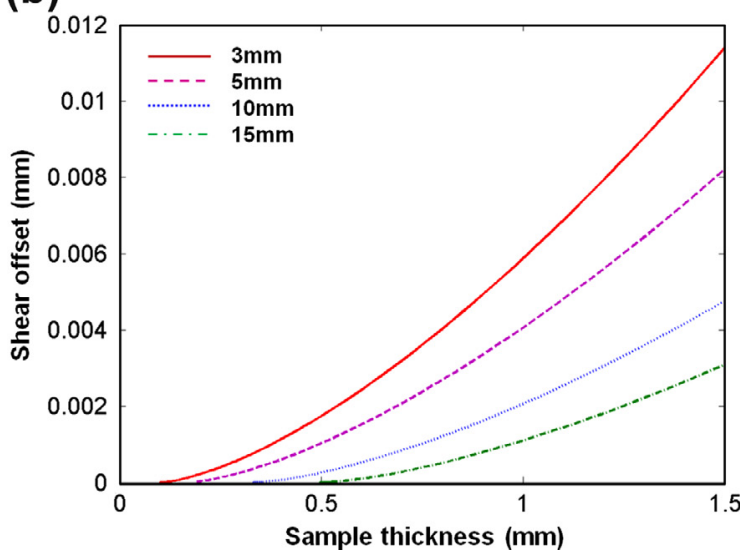

Fig. 6. (a) Shear band offset on the tensile side varies with bend curvature for different sample thicknesses; (b) Shear band offset on the tensile side varies with sample thickness for fixed bend radius $(3,5,10$, and $15 \mathrm{~mm})$.

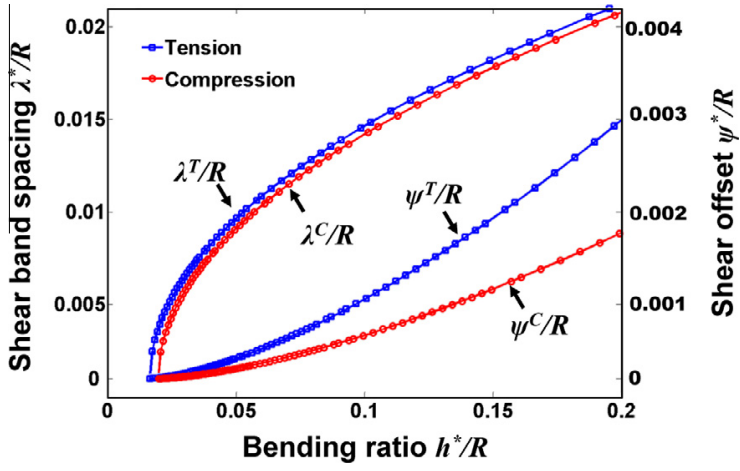

Fig. 7. Dependence of the normalized shear band spacing and shear offset on bending ratio for both tensile and compressive parts.

and its shear resistance is completely lost when $\psi^{*}=\psi_{c}^{*}$ or $\beta \Lambda^{*}=1$. When the shear offset in the mature shear band exceeds a critical value $\Delta u$, the shear band is assumed to transform into a mixed mode crack (Mode I and II) (Conner et al., 2003; Ravichandran and Molinari, 2005). Setting the shear offset $\psi^{*}$ (see Eq. (31)) equal to $\Delta u$ at $\beta \Lambda^{*}=1$, Eq. (31) can be viewed as an algebraic equation for $h_{0}^{*}$. By denoting $\varsigma^{*}=h_{0}^{*} / h^{*}$, the physically relevant solution to the cubic Eq. (31) can be expressed as

$$
\varsigma^{*}=1+\left(\left(a^{3} / 27+a^{2} / 4\right)^{1 / 2}-a / 2\right)^{1 / 3}+\left(-\left(a^{3} / 27+a^{2} / 4\right)^{1 / 2}-a / 2\right)^{1 / 3},
$$


where the non-dimensional parameter $a=\Delta u^{2} E^{\prime} D^{*} / A \sigma_{0} h^{* 2}$ and $A=12 \sigma_{0} /\left(\rho D^{*} \chi^{2} \cos ^{2} \theta^{*}\right)$. The critical strain along the axial direction at the free boundary when a crack forms in the shear band can be obtained as $\varepsilon_{c}^{*}=\varepsilon_{x x}\left(y=h^{*}\right)=\varepsilon_{y}^{*}+\varepsilon_{p}^{*}$, where $\varepsilon_{y}^{*}=\sigma_{s} /\left(D^{*} E^{\prime}\right)$ is the elastic strain at yield point and the plastic strain to fracture is expressed as

$$
\varepsilon_{p}^{*}=\Delta u^{2} /\left[A h^{* 2}\left(1-\varsigma^{*}\right)^{2}\right]
$$

See Eq. (32), $\varsigma^{*}$ is a complicated function of material properties and the sample thickness. In order to have better understanding of the ductility, the expression (33) is expanded in a Taylor series for two special cases, which is of practical interest. For large $a(\gg 1)$, corresponding to a small $h^{*}$ for a given material, the plastic strain to fracture satisfies

$$
\varepsilon_{p}^{*} \approx \Delta u^{2} /\left(A h^{* 2}\right) \text {. }
$$

For small $a(\rightarrow 0)$ or a large $h^{*}$,

$$
\varepsilon_{p}^{*} \approx \Delta u^{2} a^{-2 / 3} /\left(A h^{* 2}\right)
$$

Apparently, the size dependence of the plastic strain is not constant but varies from $h^{*-2 / 3}$ to $h^{*-2}$ with decreasing sample thickness. In smaller sample, a more significant size effect presents. Without losing the generality, the plastic strain in the tensile part is focused since it usually governs the macro-plasticity of BMGs. Here $\Delta u$ is assumed to be an order of $10 \mu \mathrm{m}$ according to Conner et al. (2003), to enable an effective comparison with their experimental results. The plastic strain to fracture in tension as a function of sample thickness for $\Delta u=10 \mu \mathrm{m}$ is depicted as a red solid line in Fig. 8. We can see that the plastic strain increases dramatically with decreasing sample thickness below a thickness of about $1 \mathrm{~mm}$ (i.e. $\varepsilon_{p}^{*} \propto h^{*-2}$ ). The strong size sensitivity of smaller sample enables a greatly enhanced plasticity by decreasing sample thickness (Chen et al., 2010; Jang et al., 2011; Song et al., 2009) . The thickness of $1 \mathrm{~mm}$ is also regarded as a ductile-to-brittle transition thickness for Vit-1 (Conner et al., 2003; Ravichandran and Molinari, 2005; Wu et al., 2011). With the further reduction of sample size, the plastic deformation keeps growing, during which a transition from localized to homogeneous deformation may occur at a critical size around hundreds nanometers (Jang et al., 2011; Volkert et al., 2008; Yoo et al., 2012). The analytical predictions show a good agreement with the experimental data for Vit-1 and other similar compositions compiled by Conner et al. (2003). On the other side, the slope of curve becomes less steep and the size sensitivity of plastic strain is weakened with sample thickness increasing. When the sample thickness is bigger than $10 \mathrm{~mm}$, minor plastic strain presents before cracking, and the size sensitivity of plasticity reaches a relatively small value, i.e. $\varepsilon_{p}^{*} \propto h^{*-2 / 3}$. This obvious transition of size dependence of plasticity in BMGs should be closely related to their intrinsic deformation behavior, i.e. shear band nucleation or propagation. To be noted that, the value of $\Delta u$ for our samples is around $50 \mu \mathrm{m}$. The plastic strain to fracture as a function of sample thickness for $\Delta u=50 \mu \mathrm{m}$ is also illustrated as a blue solid line in Fig. 8. We see that the both prediction lines show the same change trend, but the blue one for bigger $\Delta u$ presents a better capability of plastic deformation.

\section{Mechanism map of size-dependent multiple shear banding}

As for single shear banding, one considers that shear occurs simultaneously across the entire shear plane, termed shear displacement jump mechanism (Klaumünzer et al., 2011a; Maaß et al., 2011; Song et al., 2008; Song and Nieh, 2009); the other favors that shear band nucleates at a potential site and propagates progressively across the shear plane (Cheng et al., 2009; Han et al., 2009; Jiang and Dai, 2011; Jiang et al., 2008c; Liu et al., 2005a; Yang et al., 2010). The latter scenario

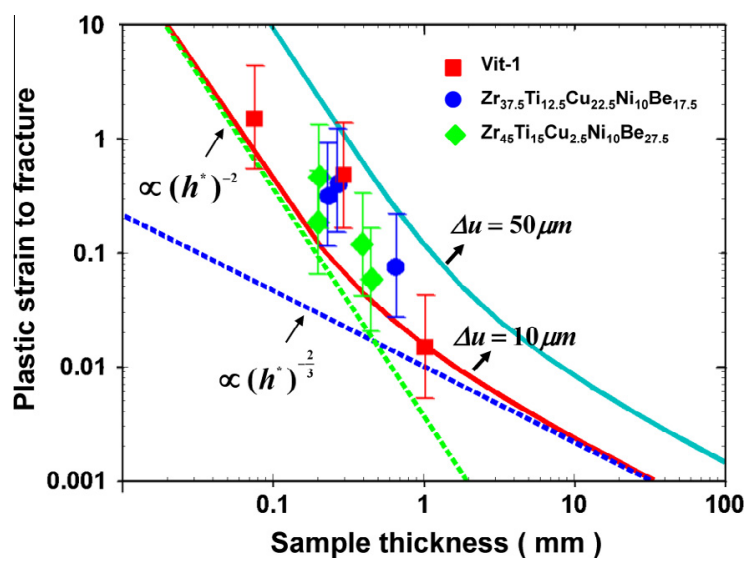

Fig. 8. Plastic strain to fracture as a function of sample thickness respectively for $\Delta u=10 \mu \mathrm{m}$ (red solid line) and $\Delta u=50 \mu \mathrm{m}$ (blue solid line).The analytical prediction is shown along with experimental data (Conner et al., 2003) for Vit-1 and other similar compositions. (For interpretation of the references to color in this figure legend, the reader is referred to the web version of this article.) 
analogous to crack propagation is found in the current experiments. Multiple shear banding is a complicated inhomogeneous deformation, as a result of collective behavior of shear band nucleation and propagation. Two fundamental and intriguing characteristics of it have been discovered: the notable scaling laws in the evolution of shear band spacing and offset (Section 4), and the inhomogeneous size effect of the accommodated plastic strain (Section 5). How to understand these phenomena? What's the underlying governing mechanism of multiple shear banding? Next, we attempt to answer these questions from the energy dissipation point of view.

As defined earlier, $E_{I}$ is the energy dissipated in the shear bands, which is contributed by shear band growth (relating to shear offset) and nucleation (relating to spacing). In the evolution process, the shear band spacing and offset would adjust to minimize the dissipation energy. The domain of interest focuses on both the tensile and compressive parts of bending. Using Eqs. (20), (30), and (31), we can derive the total dissipation energy needed to increase a unit shear offset,

$$
\Gamma_{\psi}^{*}=\frac{\partial E_{I}^{*}}{\partial \psi^{*}}=\frac{b \chi \sigma_{0} \cos \theta^{*}}{D^{*}} \sqrt{\frac{\rho\left(1-\beta \Lambda^{*} / 2\right)^{3}}{6 \beta \Lambda^{*} E^{\prime}} \cdot\left(\frac{h^{*}}{h_{0}^{*}}-1\right)},
$$

and the energy needed to decrease a unit shear band spacing,

$$
\Gamma_{\lambda}^{*}=-\frac{\partial E_{I}^{*}}{\partial \lambda^{*}}=\frac{b \chi \sigma_{0}^{2} \sin \theta^{*}}{D^{* 2}} \sqrt{\frac{\rho\left(1-\beta \Lambda^{*} / 2\right)}{24 \beta \Lambda^{*} E^{\prime 3}} \cdot\left(\frac{h^{*}}{h_{0}^{*}}-1\right)^{3}},
$$

which respectively measure the difficulty for shear band growth and nucleation. Setting $\Gamma_{\psi}^{*}=\Gamma_{\lambda}^{*}$, we obtain the critical sample thickness

$$
h_{c}^{*}=\left(1+2 \cot \theta^{*}\left(D^{*} E^{\prime} / \sigma_{0}\right)\left(1-\beta \Lambda^{*} / 2\right)\right) h_{0}^{*}
$$

At this thickness, both dissipated energy equals to

$$
\Gamma_{0}^{*}=b \chi \cos \theta^{*}\left(1-\beta \Lambda^{*} / 2\right)^{2} \cdot \sqrt{\frac{\rho \sigma_{0} \cot \theta^{*}}{3 \beta \Lambda^{*} D^{*}}}
$$

The competition of shear band nucleation and propagation can therefore illustrated by a competing map of the both dissipation energies $\Gamma_{\psi}^{*}$ and $\Gamma_{\lambda}^{*}$. The process requiring smaller dissipated energy would be easier to arise and take a dominant role.

The energy dissipated for a unit change of shear band spacing (in blue curve) or shear offset (in red curve) in dependence of the sample thickness $h^{*}$ ( for a given $h_{0}^{*}$ ) is elucidated in Fig. 9. The both energies increase monotonously with the growth of sample thickness, displaying different increasing rates. We see that, the curve for shear band growth increases faster before $h^{*}=h_{c}^{*}$, indicating that shear band propagation needs more energy to be activated. In this case, shear band nucleation would be more active than propagation, the shear band spacing would be therefore a smaller value in a thinner sample as those shown in Fig. 4. The two curves intersect at $h^{*}=h_{c}^{*}$ where the dissipation energy for the two processes both equal $\Gamma_{0}^{*}$. In this situation, shear band nucleation and growth should have the equal chance during the inhomogeneous deformation. When $h^{*}>h_{c}^{*}$, the energy dissipated for nucleation overtakes that needed for shear band growth, indicating shear band propagation would be easier to take place than nucleation. This is also reflected by the shear offset which is usually bigger in a larger sample than in a smaller one (see Fig. 6). The apparent transition from shear band nucleation to propagation is quite con-

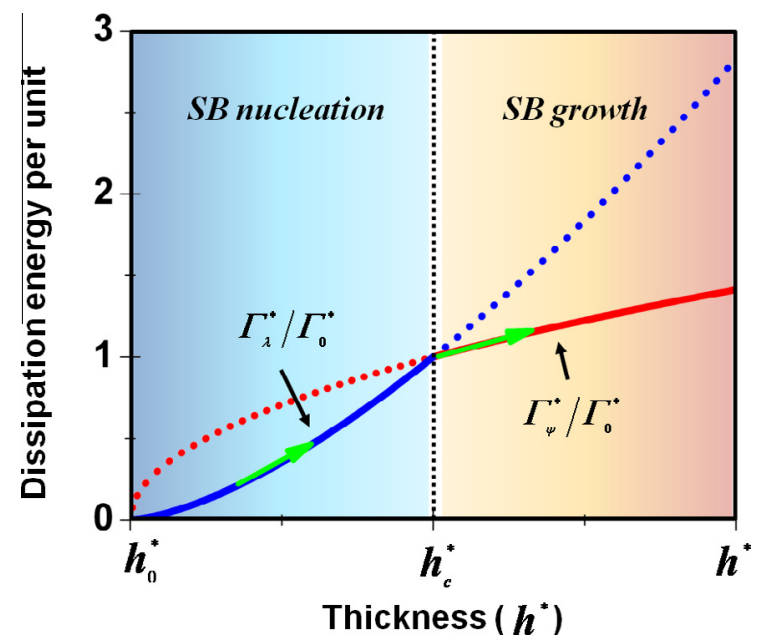

Fig. 9. Competing map of shear band (SB) nucleation and growth in dependence of sample thickness. 
sistent with the change of plastic strain with sample thickness (see Fig. 8). Compared with shear band propagation, shear band nucleation shows a stronger dependence on sample thickness (see Fig. 9). The bigger size effect of plasticity in smaller sample should be due to the stronger size sensitivity of shear band nucleation. Oppositely, when sample is big enough, the production of new shear bands would be restrained, and a propagating shear band has less opportunity to stop due to small energy required. This continuous propagation may further reduce the value of $\Gamma_{\psi}^{*}$ due to continuous free volume creation. Consequently, the nucleation of a shear band may directly lead to catastrophic failure. In this sense, the fracture of a large sample is essentially controlled by the nucleation of shear bands, as these found by previous works (Chen et al., 2010; Greer and Hosson, 2011). Whereas, the poor plastic strain and its weak size effect are mainly attributed to the fast shear band propagation. Comparing the both cases in tension and compression, we find that the transition thickness $h_{c}^{T}$ is commonly smaller than $h_{c}^{c}$ (Eq. (37)). This means that the deformation transition from shear band nucleation to propagation would first occur in tension. In another word, for a same sample, shear band nucleation may be dominant in compressive part while shear band propagation takes priority in tensile part. This is also verified by a large shear band spacing in tensile part compared with that in compressive part (Figs. 4 and 5).

\section{Concluding remarks}

Generally, a theoretical model for the evolution dynamics of multiple shear bands in BMGs has been developed under the coupling evolutions of free volume, momentum and energy. It derived the analytical solutions for shear band spacing, shear offset and critical failure strain. Following fundamental features have been revealed both by the theoretical model and a systematic experiment of a typical Zr-based BMG under in situ four-point bend tests: (i) The notable scaling laws in the evolution of shear band spacing and shear offset: shear band spacing is decreased with the increase of bend curvature, until it reaches a constant value. Meanwhile, shear offset grows with the increase of bend curvature. On the other hand, both shear band spacing and shear offset become smaller with decreasing the sample thickness. For a given curvature, the shear band spacing and offset scale respectively as the square root and the three-seconds power of thickness. (ii) The inhomogeneous size effect of plasticity: the size dependence of the failure strain is not constant but varies from $h^{*-2 / 3}$ to $h^{*-2}$ with decreasing sample thickness. To uncover their underlying physics, a competing map of shear band nucleation and propagation has been established based on energy dissipation. It is found that in a thin sample, the dissipation energy for shear band nucleation is usually smaller than that for shear band growth. Therefore, new shear bands is easier to be produced while the shear band propagation is impeded, in favor of multiple shear banding and good macro-plasticity. Moreover, the shear band nucleation presents a stronger size effect than propagation. As the two processes play different roles in samples of different dimensions, i.e., shear band nucleation dominates in small sample while shear band propagation controls plasticity in big sample, distinct size effects of plasticity display.

Our study might offer a fundamental picture of collective evolution dynamics of multiple shear bands in BMGs and provide new insights into its inherent size and pressure sensitivity. It is worth noting that the present analytical model can be used for a range of loading conditions not only the bend case. As for dynamic loading, the thermo effect on shear band evolution should appear considerable and the coupled free volume-thermo softening need to be considered, which deserves a further study in our later work.

\section{Acknowledgements}

Financial support is from the NSFC (Grants Nos. 11202221, 11132011, 11002144 and 11021262), the National Natural Science Foundation of China-NSAF Grant No: 10976100 and the National Key Basic Research Program of China (Grant Nos. 2012CB937500 and 2009CB724401).

\section{References}

Aifantis, E.C., 1987. The physics of plastic deformation. Int. J. Plast. 3, 211-247.

Aifantis, E.C., 1995. Pattern formation in plasticity. Int. J. Eng. Sci. 33, 2161-2178.

Anand, L., Su, C., 2005. A theory for amorphous viscoplastic materials undergoing finite deformations, with application to metallic glasses. J. Mech. Phys. Solids 53, 1362-1396.

Anand, L., Su, C., 2007. A constitutive theory for metallic glasses at high homologous temperatures. Acta Mater. 55, 3735-3747.

Argon, A.S., 1979. Plastic deformation in metallic glasses. Acta Metall. 27, 47-58.

Bai, Y.L., Dodd, B., 1992. Adiabatic Shear Localization. Pergamon Press, Oxford.

Batra, R.C., Lear, M.H., 2005. Adiabatic shear banding in plane strain tensile deformations of 11 thermoelastoviscoplastic materials with finite thermal wave speed. Int. J. Plast. 21, 1521-1545.

Batra, R.C., Wei, Z.G., 2006. Shear band spacing in thermoviscoplastic materials. Int. J. Impact Eng. 32, 947-967.

Bei, H., Xie, S., George, E.P., 2006. Softening caused by profuse shear banding in a bulk metallic glass. Phys. Rev. Lett. 96, 105503.

Bharathula, A., Lee, S.-W., Wright, W.J., Flores, K.M., 2010. Compression testing of metallic glass at small length scales: effects on deformation mode and stability. Acta Mater. 58, 5789-5796.

Bruhns, O.T., Schiesse, P., 1996. A continuum model of elastic-plastic materials with anisotropic damage by oritented micro voids. Eur. J. Mech. A Solids 15 , 367-396.

Burns, T.J., Davis, M.A., 2002. On repeated adiabatic shear band formation during high-speed machining. Int. J. Plast. 18, 487-506.

Cao, A.J., Cheng, Y.Q., Ma, E., 2009. Structural processes that initiate shear localization in metallic glass. Acta Mater. 57, 5146-5155.

Chaboche, J.L., 1988. Continuum damage mechanics. Part I. General concepts. J. Appl. Mech. 55, 59-64.

Chaboche, J.L., 2008. A review of some plasticity and viscoplasticiy constitutive theories. Int. J. Plast. 24, 1642-1693. 
Chen, C.Q., Pei, Y.T., De Hosson, J.T.M., 2010. Effects of size on the mechanical response of metallic glasses investigated through in situ TEM bending and compression experiments. Acta Mater. 58, 189-200.

Chen, K.W., Lin, J.F., 2010. Investigation of the relationship between primary and secondary shear bands induced by indentation in bulk metallic glasses. Int. J. Plast. 26, 1645-1658.

Chen, L., Batra, R.C., 1999. Effect of material parameters on shear band spacing in work-hardening gradient dependent thermoviscoplastic materials. Int. J. Plast. 15, 551-574.

Chen, L.Y., Fu, Z.D., Zhang, G.Q., Hao, X.P., Jiang, Q.K., Wang, X.D., Cao, Q.P., Franz, H., Liu, Y.G., Xie, H.S., Zhang, S.L., Wang, B.Y., Zeng, Y.W., Jiang, J.Z., 2008. New class of plastic bulk metallic glass. Phys. Rev. Lett. 100, 075501.

Chen, M.W., 2008. Mechanical behavior of metallic glasses: microscopic understanding of strength and ductility. Ann. Rev. Mater. Res. 38, 445-469.

Chen, Y., Jiang, M.Q., Wei, Y.J., Dai, L.H., 2011. Failure criterion for metallic glasses. Philos. Mag. 91, 4536-4554.

Cheng, Y.Q., Han, Z., Li, Y., Ma, E., 2009. Cold versus hot shear banding in bulk metallic glass. Phys. Rev. B 80, 134115.

Conner, R.D., Johnson, W.L., Paton, N.E., Nix, W.D., 2003. Shear bands and cracking of metallic glass plates in bending. J. Appl. Phys. 94, 904-911.

Conner, R.D., Li, Y., Nix, W.D., Johnson, W.L., 2004. Shear band spacing under bending of Zr-based metallic glass plates. Acta Mater. 52, $2429-2434$.

Cui, J.W., Qu, R.T., Wu, F.F., Zhang, Z.F., Shen, B.L., Stoica, M., Eckert, J., 2010. Shear band evolution during large plastic deformation of brittle and ductile metallic glasses. Philos. Mag. Lett. 90, 573-579.

Dai, L.H., 2012. Shear banding in bulk metallic glasses. In: Dodd, B., Bai, Y.L. (Eds.), Adiabatic Shear Localization: Frontiers and Advances, second ed. Elsevier, London, pp. 311-361.

Dai, L.H., Bai, Y.L., 2008. Basic mechanical behaviors and mechanics of shear banding in BMGs. Int. J. Impact Eng. 35, 704-716.

Dai, L.H., Yang, M., Liu, L.F., Bai, Y.L., 2005. Adiabatic shear banding instability in bulk metallic glasses. Appl. Phys. Lett. 87, 141916.

Das, J., Tang, M.B., Kim, K.B., Theissmann, R., Baier, F., Wang, W.H., Eckert, J., 2005. "Work-hardenable” ductile bulk metallic glass. Phys. Rev. Lett. $94,205501$.

Dodd, B., Bai, Y.L., 2012. Adiabatic Shear Localization: Frontiers and Advances, second ed. Elsevier, London.

Donovan, P.E., 1989. A yield criterion for Pd40Ni40P20 metallic glass. Acta Metall. 37, 445-456.

Dyre, J.C., Olsen, N.B., Christensen, T., 1996. Local elastic expansion model for viscous-flow activation energies of glass-forming molecular liquids. Phys. Rev. B 53, 2171-2174

Falk, M.L., Langer, J.S., 1998. Dynamics of viscoplastic deformation in amorphous solids. Phys. Rev. E 57, 7192-7205.

Flores, K.M., Dauskardt, R.H., 2001. Mean stress effects on flow localization and failure in a bulk metallic glass. Acta Mater. $49,2527-2537$.

Flores, K.M., Dauskardt, R.H., 2006. Mode II fracture behavior of a Zr-based bulk metallic glass. J. Mech. Phys. Solids 54, 2418-2435

Fornell, J., Concustell, A., Suriñach, S., Li, W.H., Cuadrado, N., Gebert, A., Baró, M.D., Sort, J., 2009. Yielding and intrinsic plasticity of Ti-Zr-Ni-Cu-Be bulk metallic glass. Int. J. Plast. 25, 1540-1559.

Gao, Y.F., 2006. An implicit finite element method for simulating inhomogeneous deformation and shear bands of amorphous alloys based on the freevolume model. Model. Simul. Mater. Sci. Eng. 14, 1329-1345.

Gao, Y.F., Wang, L., Bei, H., Nieh, T.G., 2011. On the shear-band direction in metallic glasses. Acta Mater. 59, $4159-4167$.

Gao, Y.F., Yang, B., Nieh, T.G., 2007. Thermomechanical instability analysis of inhomogeneous deformation in amorphous alloys. Acta Mater. 55, $2319-2327$.

Grady, D.E., 1992. Prosperties of an adiabatic shear-band process zone. J. Mech. Phys. Solids 40, 1197-1215.

Grady, D.E., 1994. Dissipation in adiabatic shear bands. Mech. Mater. 17, 289-293.

Grady, D.E., 2011. Adiabatic shear failure in brittle solids. Int. J. Impact Eng. 38, 661-667.

Grady, D.E., Kipp, M.E., 1987. The growth of unstable thermoplastic shear with application of steady-wave shock compression in solids. J. Mech. Phys. Solids 35, 95-118

Greer, A.L., Ma, E., 2007. Bulk metallic glasses: at the cutting edge of metals research. MRS Bull. 32, 611-619.

Greer, J.R., Hosson, J.T.M.D., 2011. Plasticity in small-sized metallic systems: intrinsic versus extrinsic size effect. Prog. Mater. Sci. 56, 654-724.

Grest, G.S., Cohen, M.H., 1981. Liquids, glasses and the glass transition: a free volume approach. Adv. Chem. Phys. 48, $455-525$.

Guo, H., Yan, P.F., Wang, Y.B., Tan, J., Zhang, Z.F., Sui, M.L., Ma, E., 2007. Tensile ductility and necking of metallic glass. Nat. Mater. 6, 735-739.

Gurson, A.L., 1977. Continuum theory of ductile rupture by void nucleation and growth. Part I. Yield criteria and flow rules for porous ductile media. Trans. ASME 99, 2-15.

Han, Z., Wu, W.F., Li, Y., Wei, Y.J., Gao, H.J., 2009. An instability index of shear band for plasticity in metallic glasses. Acta Mater. 57, $1367-1372$.

Hays, C.C., Kim, C.P., Johnson, W.L., 2000. Microstructure controllied shear band pattern formation and enhanced plasticity of bulk metallic glasses containing in situ formed ductile phase dendrite dispersions. Phys. Rev. Lett. 84, 2901-2904.

Hofmann, D.C., Suh, J.-Y., Wiest, A., Duan, G., Lind, M.-L., Demetriou, M.D., Johnson, W.L., 2008. Designing metallic glass matrix composites with high toughness and tensile ductility. Nature 451, 1085-1089.

Hsueh, C.H., Bei, H., Liu, C.T., Becher, P.F., George, E.P., 2008. Shear fracture of bulk metallic glasses with controlled applied normal stresses. Scr. Mater. 59, $111-114$.

Huang, R., Suo, Z., Prevost, J.H., Nix, W.D., 2002. Inhomogeneous deformation in metallic glasses. J. Mech. Phys. Solids 50, 1011-1027.

Jang, D.C., Gross, C.T., Greer, J.R., 2011. Effects of size on the strength and deformation mechanism in Zr-based metallic glasses. Int. J. Plast. 27, 858-867.

Jeong, H.-Y., Pan, J., 1995. A macroscopic constitutive law for porous solids with pressure-sensitive matrices and its implications to plastic flow localization. Int. J. Solids Struct. 32, 3669-3691.

Jiang, M.Q., Dai, L.H., 2007. Intrinsic correlation between fragility and bulk modulus in metallic glasses. Phys. Rev. B $76,054204$.

Jiang, M.Q., Dai, L.H., 2009. On the origin of shear banding instability in metallic glasses. J. Mech. Phys. Solids 57, 1267-1292.

Jiang, M.Q., Dai, L.H., 2011. Shear-band toughness of bulk metallic glasses. Acta Mater. 59, 4525-4537.

Jiang, M.Q., Ling, Z., Meng, J.X., Dai, L.H., 2008a. Energy dissipation in fracture of bulk metallic glasses via inherent competition between local softening and quasi-cleavage. Philos. Mag. 88, 407-426.

Jiang, M.Q., Ling, Z., Meng, J.X., Gao, J.B., Dai, L.H., 2010. Nanoscale periodic corrugation to dimple transition due to “beat” in a bulk metallic glass. Scr. Mater. $62,572-575$

Jiang, S.Y., Jiang, M.Q., Dai, L.H., Yao, Y.G., 2008b. Atomistic origin of rate-dependent serrated plastic flow in metallic glasses. Nano. Res. Lett. 3, 524-529.

Jiang, W.H., Fan, G.J., Liu, F.X., Wang, G.Y., Choo, H., Liaw, P.K., 2008c. Spatiotemporally inhomogeneous plastic flow of a bulk-metallic glass. Int. J. Plast. 24, $1-16$.

Jiang, W.H., Liu, F.X., Liaw, P.K., Choo, H., 2007. Shear strain in a shear band of a bulk-metallic glass in compression. Appl. Phys. Lett. $90,181903$.

Johnson, G.R., Cook, W.H., 1985. Fracture characteristics of three metals subjected to various strains, strain rates, temperatures and pressures. Eng. Fract. Mech. 21, 31-48.

Johnson, W., Samwer, K., 2005. A universal criterion for plastic yielding of metallic glasses with a (T/Tg)2/3 temperature dependence. Phys. Rev. Lett. 95, 195501.

Johnson, W.L., 1999. Bulk glass-forming metallic alloys: science and technology. MRS Bull. 24, 42-56.

Johnson, W.L., Lu, J., Demetriou, M.D., 2002. Deformation and flow in bulk metallic glasses and deeply undercooled glass forming liquids-a self consistent dynamic free volume model. Intermetallics 10, 1039-1046.

Khan, A.S., Liu, H., 2012a. A new approach for ductile fracture prediction on Al 2024-T351 alloy. Int. J. Plast. 35, 1-12.

Khan, A.S., Liu, H., 2012b. Strain rate and temperature dependent fracture criteria for isotropic and anisotropic metals. Int. J. Plast. $37,1-15$.

Khan, A.S., Yu, S., Liu, H., 2012. Deformation induced anisotropic responses of Ti-6Al-4V alloy. Part II. A strain rate and temperature dependent anisotropic yield criterion. Int. J. Plast. 38, 14-26.

Kim, J.-J., Choi, Y., Suresh, S., Argon, A.S., 2002. Nanocrystallization during nanoindentation of a bulk amorphous metal alloy at room temperature. Science $295,654-657$. 
Klaumünzer, D., Lazarev, A., Maaß, R., Dalla Torre, F., Vinogradov, A., Löffler, J., 2011a. Probing shear-band initiation in metallic glasses. Phys. Rev. Lett. 107, 185502.

Klaumünzer, D., Maaß, R., Löffler, J.F., 2011b. Stick-slip dynamics and recent insights into shear banding in metallic glasses. J. Mater. Res. 26, 1453-1463. Lazzeri, A., Bucknall, C.B., 1993. Dilatational bands in rubber-toughened polymers. J. Mater. Sci. 28, 6799-6808.

Lee, E.H., 1967. In: Muller, W., Shaw, M. (Eds.), Energetics III. Gordon and Breach, New York.

Lemaitre, J., 1985. A continuous damage mechanics model for ductile fracture. J. Eng. Mater. Technol. 107, 83-89.

LeRoy, G., Embury, J., Edwards, G., Ashby, M.F., 1981. A model of ductile fracture based on the nucleation and growth of voids. Acta Metall. 29, $1509-1522$. Lewandowski, J.J., Greer, A.L., 2006. Temperature rise at shear bands in metallic glasses. Nat. Mater. 5, 15-18.

Li, J., Spaepen, F., Hufnagel, T.C., 2002. Nanometer-scale defects in shear bands in a metallic glass. Philos. Mag. A 82, 2623-2630.

Liu, L.F., Dai, L.H., Bai, Y.L., Wei, B.C., 2005a. Initiation and propagation of shear bands in Zr-based bulk metallic glass under quasi-static and dynamic shear loadings. J. Non-Cryst. Solids 351, 3259-3270.

Liu, L.F., Dai, L.H., Bai, Y.L., Wei, B.C., Eckert, J., 2005b. Behavior of multiple shear bands in Zr-based bulk metallic glass. Mater. Chem. Phys. 93, $174-177$.

Liu, Y.H., Wang, G., Wang, R.J., Zhao, D.Q., Pan, M.X., Wang, W.H., 2007. Super plastic bulk metallic glasses at room temperature. Science 315, $1385-1388$.

Lu, J., Ravichandran, G., Johnson, W.L., 2003. Deformation behavior of the Zr41.2Ti13.8Cu12.5Ni10Be22.5 bulk metallic glass over a wide range of strainrates and temperatures. Acta Mater. 51, 3429-3443.

Maaß, R., Klaumünzer, D., Löffler, J.F., 2011. Propagation dynamics of individual shear bands during inhomogeneous flow in a Zr-based bulk metallic glass. Acta Mater. 59, 3205-3213.

McClintock, F.A., Kaplan, S.M., Berg, C.A., 1966. Ductile fracture by hole growth in shear bands. Int. J. Fract. Mech. 2, 614-627.

Meyers, M.A., 1994. Dynamic Behavior of Materials. John Wiley, New York.

Meyers, M.A., Nesterenko, V.F., LaSalvia, J.C., Xue, Q., 2001. Shear localization in dynamic deformation of materials: microstructural evolution an selforganization. Mater. Sci. Eng. A 317, 204-225.

Meyers, M.A., Xu, Y.B., Xue, Q., Pérez-Prado, M.T., McNelley, T.R., 2003. Microstructural evolution in adiabatic shear localization in stainless steel. Acta Mater. $51,1307-1325$.

Miracle, D.B., Concustell, A., Zhang, Y., Yavari, A.R., Greer, A.L., 2011. Shear bands in metallic glasses: size effects on thermal profiles. Acta Mater. 59, 28312840.

Molinari, A., 1997. Collective behavior and spacing of adiabatic shear bands. J. Mech. Phys. Solids 45, 1551-1575.

Molinari, A., Musquar, C., Sutter, G., 2002. Adiabatic shear banding in high speed machining of Ti-6Al-4V: experiments and modeling. Int. J. Plast. 18, 443459.

Mott, N.F., 1947. Fragmentation of Shell Cases. Proc. R. Soc. London 189, 300-308.

Murakami, S., Ohno, N., 1981. A continuum theory of creep and creep damage. In: Ponter, A.R.S., Hayhurst, D.R. (Eds.), Creep in Structures. Springer, Berlin, pp. 422-444.

Needleman, A., Tvergaard, V., 1984. An analysis of ductile rupture in notched bars. J. Mech. Phys. Solids 32, 461-490.

Nesterenko, V.F., Meyer, M.A., Wright, T.W., 1998. Self-organization in the initiation of adiabatic shear bands. Acta Mater. 46, 327-340.

Ott, R.T., Sansoz, F., Jiao, T., Warner, D., Fan, C., Molinari, J.F., Ramesh, K.T., Hufnagel, T.C., 2006. Yield criteria and strain-rate behavior of $\mathrm{Zr}_{57.4} \mathrm{Cu}_{16.4} \mathrm{Ni}_{8.2} \mathrm{Ta}_{8} \mathrm{Al}_{10}$ metallic-glass-matrix composites. Metall. Mater. Trans. A 37, 3251-3257.

Pan, D., Inoue, A., Sakurai, T., Chen, M.W., 2008. Experimental characterization of shear transformation zones for plastic flow of bulk metallic glasses. PNAS $105,14769-14772$.

Ravichandran, G., Molinari, A., 2005. Analysis of shear banding in metallic glasses under bending. Acta Mater. 53, 4087-4095.

Rice, J.R., Tracey, D.M., 1969. On the ductile enlargement of voids in triaxial stress fields. J. Mech. Phys. Solids 17, 201-217.

Ruan, H.H., Zhang, L.C., Lu, J., 2011. A new constitutive model for shear banding instability in metallic glass. Int. J. Solids Struct. 48, $3112-3127$.

Schuh, C., Hufnagel, T., Ramamurty, U., 2007. Mechanical behavior of amorphous alloys. Acta Mater. 55, 4067-4109.

Schuh, C.A., Lund, A.C., 2003. Atomistic basis for the plastic yield criterion of metallic glass. Nat. Mater. 2, 449-452.

Sergueeva, A.V., Mara, N.A., Kuntz, J.D., Lavernia, E.J., Mukherjee, A.K., 2005. Shear band formation and ductility in bulk metallic glass. Philos. Mag. 85, 26712687.

Song, S.X., Bei, H., Wadsworth, J., Nieh, T.G., 2008. Flow serration in a Zr-based bulk metallic glass in compression at low strain rates. Intermetallics $16,813-$ 818.

Song, S.X., Lai, Y.H., Huang, J.C., Nieh, T.G., 2009. Homogeneous deformation of Au-based metallic glass micropillars in compression at elevated temperatures. Appl. Phys. Lett. 94, 061911.

Song, S.X., Nieh, T.G., 2009. Flow serration and shear-band viscosity during inhomogeneous deformation of a Zr-based bulk metallic glass. Intermetallics 17 , $762-767$.

Spaepen, F., 1977. A microscopic mechanism for steady state inhomogeneous flow in metallic glases. Acta Metall. 25, 407-415.

Spaepen, F., 1981. Defects in amorphous metals. In: Balian, R. et al. (Eds.), Physics of Defects, Les Houches Lectures, vol. XXXV. North-Holland, Amsterdam, pp. 133-174.

Steif, P.S., Spaepen, F., Hutchinson, J.W., 1982. Strain localization in amorphous metals. Acta Matall. 30, 447-455.

Subhash, G., Zhang, H., 2007. Shear band patterns in metallic glasses under static indentation, dynamic indentation, and scratch processes. Matall. Mater. Trans. A 38, 2936-2942.

Sun, B.A., Yu, H.B., Jiao, W., Bai, H.Y., Zhao, D.Q., Wang, W.H., 2010a. Plasticity of ductile metallic glasses: a self-organized critical state. Phys. Rev. Lett. 105, 035501.

Sun, L., Jiang, M.Q., Dai, L.H., 2010b. Intrinsic correlation between dilatation and pressure sensitivity of plastic flow in metallic glasses. Scr. Mater. 63, 945948.

Thamburaja, P., 2011. Length scale effects on the shear localization process in metallic glasses: a theoretical and computational study. J. Mech. Phys. Solids 59, 1552-1575.

Thamburaja, P., Ekambaram, R., 2007. Coupled thermo-mechanical modelling of bulk-metallic glasses: theory, finite-element simulations and experimental verification. J. Mech. Phys. Solids 55, 1236-1273.

Trexler, M.M., Thadhani, N.N., 2010. Mechanical properties of bulk metallic glasses. Prog. Mater. Sci. 55, 759-839.

Tvergaard, V., 1981. Influence of voids on shear band instabilbities under plane strain conditions. Int. J. Fract. 17, 389-407.

Tvergaard, V., 1982. On localization in ductile materials containing spherical voids. Int. J. Fract. 18, 237-252.

Volkert, C.A., Donohue, A., Spaepen, F., 2008. Effect of sample size on deformation in amorphous metals. J. Appl. Phys. 103, 083539

Voyiadjis, G.Z., Kattan, P.I., 1992. A plasticity-damage theory for large deformations of solids. I Theoretical formulation. Int. J. Eng. Sci. 30, 1089-1108.

Voyiadjis, G.Z., Kattan, P.I., 2005. Damage Mechanics. Taylor \& Francis, Boca Raton.

Wang, W.H., 2012. The elastic properties, elastic models and elastic perspectives of metallic glasses. Prog. Mater. Sci. 57, 487-656.

Wen, J., Huang, Y., Hwang, K.C., Liu, C., Li, M., 2005. The modified Gurson model accounting for the void size effect. Int. J. Plast. 21, 381-395.

Wright, T.W., 2002. The Physics and Mathematics of Adiabatic Shear Bands. Cambridge University Press, Cambridge.

Wright, T.W., Ockendon, H., 1996. A scaling law for the effect of inertia on the formation of adiabatic shear bands. Int. J. Plast. 12, 927-934.

Wright, W.J., Samale, M.W., Hufnagel, T.C., LeBlanc, M.M., Florando, J.N., 2009. Studies of shear band velocity using spatially and temporally resolved measurements of strain during quasistatic compression of a bulk metallic glass. Acta Mater. 57, 4639-4648.

Wright, W.J., Schwarz, R.B., Nix, W.D., 2001. Localized heating during serrated plastic flow in bulk metallic glasses. Mater. Sci. Eng. A 319-321, 229-233.

Wu, F.F., Zheng, W., Wu, S.D., Zhang, Z.F., Shen, J., 2011. Shear stability of metallic glasses. Int. J. Plast. 27, 560-575.

Xie, S., George, E.P., 2008. Hardness and shear band evolution in bulk metallic glasses after plastic deformation and annealing. Acta Mater. 56, 5202-5213. 
Xu, Y.B., Meyers, M.A., 2012. Nanostructural and microstructural aspects of shear localization at high-strain rates for materials. In: Dodd, B., Bai, Y.L. (Eds.), Adiabatic Shear Localization, second ed. Elsevier, London.

Xue, Q., Meyers, M.A., Nesterenko, V.F., 2002. Self-organization of shear bands in titanium and Ti-6Al-4V alloy. Acta Mater. 50, 575-596.

Xue, Q., Meyers, M.A., Nesterenko, V.F., 2004. Self organization of shear bands in stainless steel. Mater. Sci. Eng. A 384, 35-46.

Yang, B., Morrison, M.L., Liaw, P.K., Buchanan, R.A., Wang, G., Liu, C.T., Denda, M., 2005. Dynamic evolution of nanoscale shear bands in a bulk-metallic glass. Appl. Phys. Lett. 86, 141904.

Yang, Q., Mota, A., Ortiz, M., 2006. A finite-deformation constitutive model of bulk metallic glass plasticity. Comput. Mech. 37, $194-204$.

Yang, Y., Ye, J.C., Lu, J., Liaw, P.K., Liu, C.T., 2010. Characteristic length scales governing plasticity/brittleness of bulk metallic glasses at ambient temperature. Appl. Phys. Lett. 96, 011905.

Yao, K.F., Ruan, F., Yang, Y.Q., Chen, N., 2006. Superductile bulk metallic glass. Appl. Phys. Lett. 88, 122106.

Ye, G.G., Xue, S.F., Jiang, M.Q., Tong, X.H., Dai, L.H., 2013. Modeling periodic adiabatic shear band evolution during high speed machining Ti-6Al-4V alloy. Int. J. Plast. 40, 39-55.

Yoo, B.-G., Kim, J.-Y., Kim, Y.-J., Choi, I.-C., Shim, S., Tsui, T.Y., Bei, H., Ramamurty, U., Jang, J.-i., 2012. Increased time-dependent room temperature plasticity in metallic glass nanopillars and its size-dependency. Int. J. Plast. 37, 108-118.

Zbib, H.M., Aifantis, E.C., 1988. On the localization and postlocalization behavior of plastic deformation I/II/II. Res. Mech. 23, $261-305$.

Zbib, H.M., Aifantis, E.C., 1992. On the gradient-dependent theory of plasticity and shear banding. Acta Mech. 92, $209-225$.

Zbib, H.M., Jubran, J.S., 1992. Dynamic shear banding -a 3-dimensional analysis. Int. J. Plast. 8, 619-641.

Zhang, H., Maiti, S., Subhash, G., 2008. Evolution of shear bands in bulk metallic glasses under dynamic loading. J. Mech. Phys. Solids 56, 2171-2187.

Zhang, H.W., Jing, X.N., Subhash, G., Kecskes, L.J., Dowding, R.J., 2005. Investigation of shear band evolution in amorphous alloys beneath a Vickers indentation. Acta Mater. 53, 3849-3859.

Zhao, P., Li, J., Wang, Y., 2012. Heterogeneously randomized STZ model of metallic glasses: softening and extreme value statistics during deformation. Int. J. Plast. 40, 1-22.

Zhou, F., Wright, T.W., Ramesh, K.T., 2006a. The formation of multiple adiabatic shear bands. J. Mech. Phys. Solids 54, 1376-1400.

Zhou, F., Wright, T.W., Ramesh, K.T., 2006b. A numerical methodology for investigating the formation of adiabatic shear bands. J. Mech. Phys. Solids 54, 904926. 